\title{
Some Aspects on the Modelling of Microstructure Deformation Mechanisms with Gradient Truss Model (GTM) Bar Elements
}

\section{Olufemi T. Akintayo ${ }^{a}$}

aLaboratory of Mechanics and Materials, Department of Civil Engineering, School of Engineering, Aristotle University of Thessaloniki, GR-54124, Greece. E-mail: femiakint@reclomat.com

\section{*Corresponding author}

http://dx.doi.org/10.1590/1679-78256007

\begin{abstract}
Unlike the classical truss model, the constitutive equation of the gradient truss model (GTM) earlier presented by the author introduces higher order strain gradient terms and a characteristic internal length parameter: Thus, considering the interaction between macroscopic and microscopic length scales in the constitutive response. Extra non-classical boundary conditions are required to solve the governing equation. In this paper, the microstructure of a material is defined in a simple manner by representing three typical underlying phenomena based on the spatial variation of strain and three combinations of extra non-classical boundary conditions of the derivative of displacement (strain) and higher order derivative of displacement (strain gradient) imposed at the bar support. To quantify the imposed strain gradient at the bar support, a simple relation is derived. Consequently, the influence of strain, strain gradient and the characteristic internal length parameter at the microstructure during deformation is readily captured and the three underlying phenomena qualitatively modelled by the three GTM bar elements. In addition, strengthening and weakening mechanisms in deformation are revealed. Numerical examples are presented as illustration.
\end{abstract}

\section{Keywords}

Gradient truss model bar elements, Characteristic internal lengths, Strain gradient, Controlling microstructure feature.

\section{Graphical Abstract}

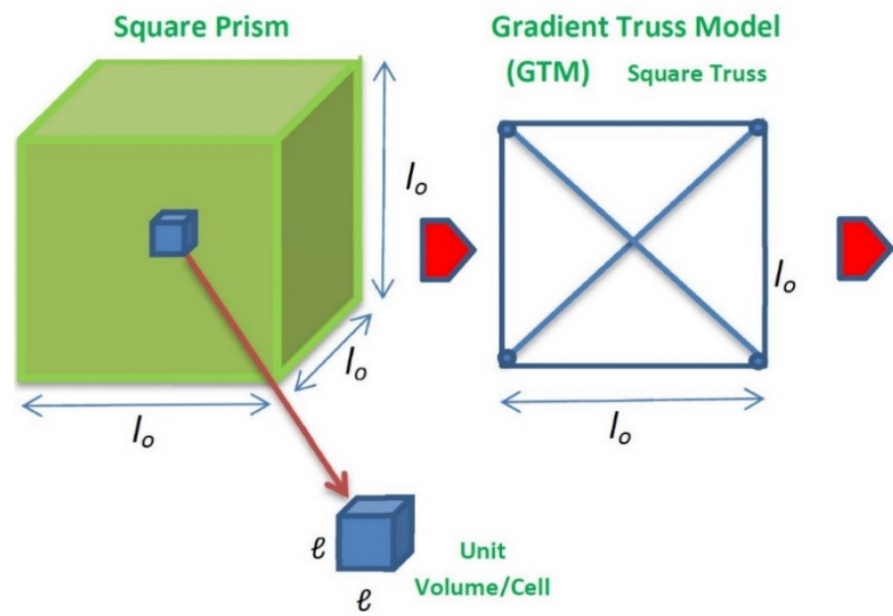

GTM Bar Element

Types

GTM BE 1

(Homogeneous)

GTM BE 2

(Heterogeneous

Strong Domain)

GTM BE 3

(Heterogeneous

Weak Domain) 


\section{INTRODUCTION}

Most material composition exhibits some kind of order at one or several spatial scales. For instance in the perfect order of the crystal lattice in a metal or the imperfect order in the arrangement of fibres and matrix in fibre-reinforced laminate, a number of spatial scales can be identified and distinguished with size and spacing having a typical order of magnitude Uchic et al. (2004), Clemens et al. (2017). Hence a particular controlling microstructural feature and a characteristic internal length can be readily identified Greer and De Hosson (2011), Isasti et al. (2014).

In general, continuum theories can present relevant approximate response of a material cost effectively. However, between the atomic length scale and that of the engineering structure or component they are insufficient in the representation of particular scales of interest. Consequently continuum theories that can predict the average response of the real size components and also take into account the effect of the typical scale of the microstructure are required. The concept of average in this case is usually interpreted as "ensemble average" since volume averaging may smoothen stress and strain variations to an unacceptable degree Peerlings and Fleck (2004). The classical elasticity or plasticity theories are not sufficiently high spatial order for the strain field and do not possess a structure amenable to non-trivial material stability analysis. Moreover the only material length implicitly involved in these field equations is the interatomic distance or lattice parameter and it is not physically reasonable to appeal to this length directly for explaining the overabundance of deformation patterns observed at the various scales ranging from nanometers (dislocations) and micrometer (slip bands) to millimetres (shear bands) Aifantis (1992).

Closed form macroscopic continuum framework which take into account microstructure effect and particularly the relevant length scale of the microstructure includes the works of Cosseret and Cosserat (1909), Toupin (1962), Mindlin (1964), Kroner (1967), Ben-Amoz (1976) and Aifantis (1984). By assuming relatively simple constitutive relations at dominant size scale, microstructure effects can be described naturally and in qualitative details. Strain gradient models introduce explicit gradients of strain measures (plastic or internal elastic strain) in the evolution equation. More recent studies include: Polizzotto (2010), Askes and Aifantis (2011), Bertram (2015), Lazar (2016), Umesh et al. (2017), Beheshti (2017). With the inclusion of a characteristic length (i.e. material parameter with internal length scale), the interaction between macroscopic and microscopic length scales is considered in the constitutive response. Hence displacement at the macro-scale and micro-scale can be identified. For the gradient elasticity study of a bar, additional useful information not provided by the classical equivalent has been observed: Altan and Aifantis (1997), Gao et al. (1999) and Tsepoura et al. (2002).

On the other hand, to computationally study the mechanical behavior of materials a first choice is the standard Finite Element Method (FEM) with complex elements: While it allows the computational stress analysis of a continuum with any boundary conditions and loading, several problems and complications arise mainly due to the inclusion of a nonlinear constitutive law when updating the stiffness matrix of the finite element Argyris $(1978,1981,1994)$. Whereas with the classical truss models, the bars of the truss are the simplest possible finite elements that can be used for the analysis of a continuum in a simple manner: Bazant (1997), Geol et al. (1997), Papadopoulos and Xenidis (1998) and Akintayo et al. $(1998,2000)$. Through the assumed nonlinear uniaxial stress-strain $(\sigma-\varepsilon)$ laws for the bars, the effect of biaxial or triaxial physical nonlinearity of a continuum can easily be incorporated. In addition, by updating the nodal coordinates of the truss and writing the equilibrium conditions with respect to the deformed configuration within each step of a static incremental loading, the geometric nonlinearity and associated structural instability can also be described.

In search of an efficient and cost effective computational tool that considers the influence of microstructure in a very simple manner, Akintayo (2011) and Akintayo et al. (2012) first presented the gradient truss model (GTM) bar elements. By incorporating the phenomenological gradient elastic theory of Aifantis $(1984,1992)$ into the simple numerical process of the classical elasticity truss model, higher order strain gradient terms and an internal length parameter were included in the elastic constitutive stress-strain relation of a representative GTM bar element: Thus the microstructure deformation response is captured. Subsequently, Akintayo (2014a) obtained several bar displacementforce relations for different combinations of extra non-classical boundary conditions of the derivative of displacement (strain) and higher order derivative of displacement (strain gradient) imposed at the bar support. The corresponding stiffness matrices for the truss bar elements were presented in Akintayo (2018).

Generally, the behaviour of the GTM bar element was shown to depend on the ratio between the bar length and the characteristic internal length and the boundary conditions. It was also observed that bars with imposed strain or imposed strain gradient at the unconstrained boundary were less stiff, while those with imposed strain gradient at the constrained boundary resulted in stiffer bar responses Akintayo (2014a, 2018). However, these preliminary works were inconclusive on the implication of these observations and the possible application of the bar elements.

Therefore, this paper addresses some of these aspects necessary towards modelling with the GTM bar elements. Firstly, a description of the GTM in relation to a block of material and a discussion on the characteristic internal length is 
presented: Begley and Hutchinson (1998); Shu and Fleck (1998); Nix and Gao (1998); McElhaney et al. (1998); Yuan and Chen (2001); Stolken and Evans (1998); Anderson and Lakes (1994); Lakes (1995) and Geers et al. (1999). Secondly, the GTM bar element field parameters are presented and six combinations of non-classical boundary conditions imposed at the bar supports are re-defined explicitly in relation to spatial variation of strain in the block: Thus, three underlying phenomena at the microstructure are represented by three GTM bar elements (GTM BEs). Thirdly, to address the need to qualitatively determine the characteristic internal lengths for use in GTM, a simple statistical-deterministic approach is presented as illustration. Fourthly, to qualitatively quantify the imposed strain gradient at the bar support it is related to the characteristic internal length and a deformation parameter in a simple manner. Based on the aforementioned, numerical illustrations showing the behaviour of these relations and modelling with the three GTM bar elements are presented: Thus, the influence of strain, strain gradient and the microstructure parameter in deformation is revealed. Finally, the modelling considerations for a short glass fibre-reinforced polypropylene (SGFPP) Geers et al. (1999) and a porous metal fiber/powder sintered composite sheet (PMFPSCS) Zou et al. (2016) are studied in relation to strengthening and weakening deformation mechanisms.

\section{GRADIENT TRUSS MODEL DESCRIPTION AND FIELD PARAMETERS}

\subsection{Gradient Truss Model (GTM) Representation of the Microstructure}

Similar to the classical elastic truss model in Papadopoulos and Xenidis (1998) and Akintayo et al. $(1998,2000)$, the proposed gradient truss model simulates a material block by a plane truss consisting of a coarse grid of equal elementary square trusses including both of the diagonal bars Akintayo (2011) and Akintayo et al. (2012). The relation between the strains and stresses of the bars connected to an interior node of the truss model and the strain and stress states at a point of the continuum are represented in Figure 1 by: $\varepsilon_{\mathrm{x}}=\left(\varepsilon_{\mathrm{r}}+\varepsilon_{\mathrm{l}}\right) / 2 ; \sigma_{\mathrm{x}}=\left(\sigma_{\mathrm{r}}+\sigma_{\mathrm{l}}\right) / 2 ; \varepsilon_{\mathrm{y}}=\left(\varepsilon_{\mathrm{a}}+\varepsilon_{\mathrm{u}}\right) / 2 ; \sigma_{\mathrm{y}}=\left(\sigma_{\mathrm{a}}+\sigma_{\mathrm{u}}\right) / 2$; $\gamma_{\mathrm{xy}}=\left(\varepsilon_{\mathrm{ra}}-\varepsilon_{\mathrm{la}}+\varepsilon_{\mathrm{lu}}-\varepsilon_{\mathrm{ru}}\right) \sin 45^{\circ} / 4 ; \tau_{\mathrm{xy}}=\left(\sigma_{\mathrm{ra}}-\sigma_{\mathrm{la}}+\sigma_{\mathrm{lu}}-\sigma_{\mathrm{ru}}\right) \sin 45^{\circ} / 4$. As shown in Figure 1 the determination of the bar cross sections of the truss model reduces to the simulation of a square prism under biaxial stress by an elementary square truss and the square prism could be related to a representative volume of the material Akintayo (2011).

The relation between the continuum theory and the classical truss model depends on the degree of discretization employed. As the discretization gradually becomes more detailed (finer), the strain and stress fields of the continuum will gradually describe the microstructure behavior more accurately. However, the computational cost also increases. Since the goal of the GTM is to model the microstructure in a simple and cost effective manner, the GTM bar elements are distinguished from the classical elastic bar elements by their ability to support elastic strain gradients along the bar; hence, the phenomenological theory of gradient elasticity proposed by Aifantis $(1984,1992)$ is applied.

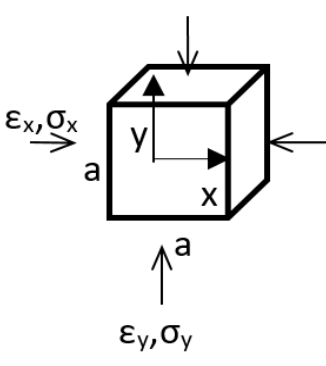

(a)

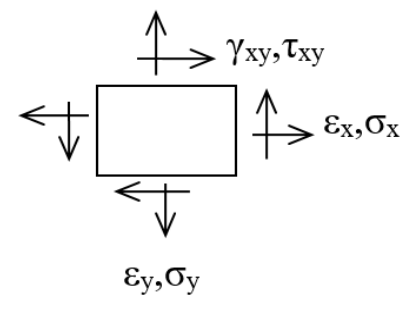

(b)

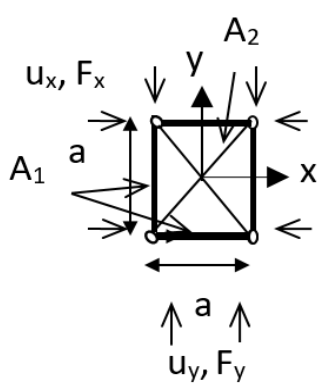

(c)

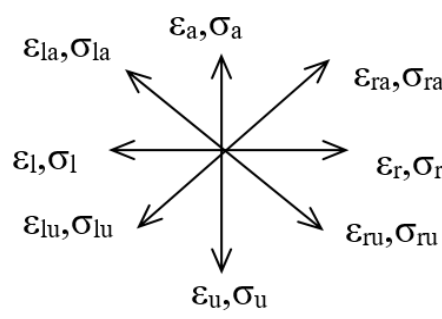

(d)

Figure 1. (a) Square prism under biaxial stress; (b) Stress and strain at a point of the continuum; (c) Elementary plane square truss model; (d) Stress and strain state at truss node

For the constitutive equation of the representative volume of Figure 2, the deformation of a unit volume is incorporated as higher order strain gradients. Thus, the deformation of the bar element at each node not only considers macro deformation at that point, but also the micro deformation in relation to the unit volumes within that neighbourhood local representative square prism. 


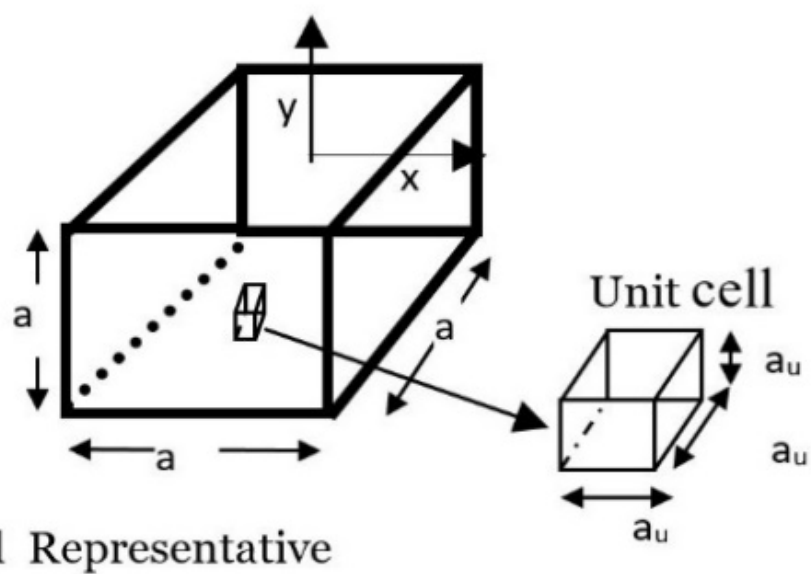

\section{Local Representative}

Figure 2. (a) Local representative volume containing unit cell

Mindlin (1964) showed that the material local representative volume contains material particles and embedded in each material particle is assumed to be the micro volume, the unit cell. The local representative volume could be a polymer, a polycrystal or a granular material and their unit volume (cell) a molecule, a crystalline or a grain respectively. Hence, by isolating a typical unit cell element from the grid of say a crystal lattice (local representative volume), the modelling of a continuum with micro deformation can be developed. Nevertheless, heterogeneous materials with complex microstructures create a challenge in the modelling of their effective behavior. Volume fractions, crystallographic and morphologic orientations but also sizes and shapes of the grains and particles are constitutive elements of the microstructure of heterogeneous materials which have to be tailored. Ben-Amoz (1976) showed that the particulate can be represented as the unit volume of a heterogeneous medium consisting of inclusions of arbitrary geometry embedded in a matrix material. Consequently, the effects of inclusion arrangement on the macro-behaviour are entirely contained in a geometrical constant, which must be evaluated from given information on the distribution and shape of the inclusions in a local volume of finite dimensions. Though the treatment is deterministic, there is an intimate connection between the local representative volume of the irregular and regular cells and the related equating ensemble averages to local volume averages arising in statistical theories of inhomogeneous media Beran and McCoy (1970).

As will be addressed in the next section, the characteristic internal length $(\ell=\sqrt{ } c$ where $c$ is the gradient elastic coefficient) of the material is seen as the microstructure parameter that scales the strain gradient effect. Hence, in a simple manner it could be related to the dimension of a unit volume from available experimental or statistical deterministic data of the material. Akintayo (2014d) showed that the behavior of GTM depends on the ratio $\gamma=I_{o} / \ell$ between the GTM bar element length $l_{o}$ and the characteristic internal length $\ell$.

\subsection{The Characteristic Internal Length}

The characteristic internal length parameter can be seen as the scale correction term for the material behaviour law. When the material deformation occurs at a certain scale they can differ from the bulk behaviour of the material. The length scale parameter captures this difference in material behaviour in the constitutive equation. Invariably when considering the microstructure deformation mechanism the gradient-dependent behavior is expected to play an important role once the length scale associated with the local deformation gradients become sufficiently large in comparison to the controlling microstructural feature (e.g., mean spacing between inclusions relative to the inclusion size when considering a microstructure with dispersed inclusions, size of the plastic process zone at the front of the crack tip, the mean spacing between dislocations, the grain size, etc) Geers et al. (1999).

Experimentally several authors have studied strain gradient dominated deformation and the characteristic internal length: Bazant and Pijaudier-Cabot (1989), Fleck et al (1994); Anderson \& Lakes (1994); Lakes (1995); Gao et al (1999); Geers et al. (1999); Begley and Hutchinson (1998); Shu and Fleck (1998); Nix and Gao (1998); McElhaney et al. (1998); Yuan and Chen (2001); Stolken and Evans (1998); Lam et al. (2003); Abu Al-Rub and Voyiadjis (2004). See Table 1: a list of some characteristic internal lengths obtained for different materials. 
Table 1: Material Characteristic internal length and properties from literature

\begin{tabular}{|c|c|c|c|c|}
\hline \multicolumn{5}{|c|}{ Material Properties } \\
\hline Material \& Biography & $\begin{array}{l}\text { Characteristic Internal } \\
\text { Length }\end{array}$ & $\begin{array}{l}\text { Particle/fibre } \\
\text { cell/size }\end{array}$ & Strain & Young's Modulus \\
\hline $\begin{array}{l}\text { M1. Fleck et al. (1994) microtorsion test of thin } \\
\text { copper wires }\end{array}$ & $\ell=4 \mu \mathrm{m}$ & & & \\
\hline M2. Stolken and Evans (1998) for nickel & $\ell=5 \mu \mathrm{m}$ & & & $190-220 \mathrm{GPa}$ \\
\hline $\begin{array}{l}\text { M3. Yuan and Chen (2001) for polycrystal } \\
\text { copper }\end{array}$ & $\ell=6 \mu \mathrm{m}$ & & & $111 \mathrm{GPa}$ \\
\hline $\begin{array}{l}\text { M4. Yuan and Chen (2001) for single crystal } \\
\text { copper. }\end{array}$ & $\ell=20 \mu \mathrm{m}$ & & & 67-192 GPa \\
\hline $\begin{array}{l}\text { M5. Nix and Gao (1998) Annealed single crystal } \\
\text { copper }\end{array}$ & $\ell=12 \mu \mathrm{m}$ & & & 67-192 GPa \\
\hline $\begin{array}{l}\text { M6. Nix and Gao (1998) Coldworked } \\
\text { polycrystalline copper. }\end{array}$ & $\ell=5.84 \mu \mathrm{m}$ & & & $111 \mathrm{GPa}$ \\
\hline $\begin{array}{l}\text { M7. Anderson \& Lakes (1994) Closed cell } \\
\text { polyurethane foam }\end{array}$ & $\ell=0.33 \mathrm{~mm}$ & $0.05-0.15 \mathrm{~mm}$ & & $300 \mathrm{MPa}$ \\
\hline $\begin{array}{l}\text { M8. Lakes (1995) polymethacrylamide closed cell } \\
\text { foam }\end{array}$ & $\begin{array}{l}\ell_{t}=0.80 \mathrm{~mm} \\
\ell_{b}=0.77 \mathrm{~mm}\end{array}$ & $0.65 \mathrm{~mm}$ & & $637 \mathrm{MPa}$ \\
\hline $\begin{array}{l}\text { M9. Zou et al. (2016) PMFPSCS 80\% \& 90\% } \\
\text { porosity }\end{array}$ & $\begin{array}{l}\text { Evaluated } \ell=1.0-2.0 \\
\qquad \mathrm{~mm}\end{array}$ & $\begin{array}{l}\text { Copper fibre size } \\
15.0 \mathrm{~mm}\end{array}$ & $\begin{array}{l}0.05 \%- \\
0.08 \% .\end{array}$ & $3700 \mathrm{MPa}$ \\
\hline M10. Geers et al. (1999) SGFPP & $\ell=1-2.236 \mathrm{~mm}$ & $\begin{array}{l}\text { Glass fibre size } \\
12.5 \mathrm{~mm}\end{array}$ & $1.1 \%$ & $3200 \mathrm{MPa}$ \\
\hline
\end{tabular}

However, the definition of the characteristic internal length is not unambiguous and depends on the constitutive framework in which it is embedded. Specific experimental procedures and methods have to be set up in agreement with the physical phenomena which govern the deformation process. The necessary data have to be extracted from complex tests combined with an iterative procedure based on a proper understanding of the significance and influence of each parameter Geers et al. (1999). In addition, it has been shown that the material properties of gradient theories cannot be effectively determined by using typical tensile test where uniform deformation is encountered since gradient-based models hinges on the ability to determine the constitutive length parameter that scales the gradient effect Abu Al-Rub and Voyiadjis (2004). Hence strain gradient dominated experiments are required in order to achieve any useful results. The study of Begley and Hutchinson (1998) and Shu and Fleck (1998) indicated that indentation experiments might be the most effective test for measuring the length-scale parameter $\ell$. It has also been shown that a constant value of the material length scale is not always realistic and that different problems could require different values Tsagrakis and Aifantis, (2002). This change in length-scale magnitude is physically sound since there is a continuous modification of the material characteristics with time.

Though several parameters contribute to the determined characteristic internal length, however a simple but noteworthy observation from Table 1 shows it falls within a scale range for most materials. For instance, different values were obtained for copper samples based on the different tests carried out, but these values fall between 4-20 $\mu \mathrm{m}$ within the micrometer length scale which is the scale of the grain size of copper.

\subsection{Gradient Truss Model (GTM) Bar Element}

As part of this section the formulation of the field relations of the GTM is addressed. Like in the classical elasticity case, to obtain the field relations for the bar element of Figure $3 \mathrm{a}$, the elastic bar with length $I_{0}$ shown in Figure $3 \mathrm{~b}$ is considered fixed (constrained) at one end and subject to an axial tensile force $F$ at the free (unconstrained) right end $\mathrm{x}=I_{\text {o }}$. The modulus of elasticity is $\mathrm{E}$, while the cross-sectional area is $\mathrm{A}$.

The force and displacements at the node are given as $f_{j i}=-f_{i i}$ and $u_{j i}=u_{i i}$. However, here the bar is assumed to be able to support elastic strain gradients along the bar; hence, the special theory of gradient elasticity proposed by Aifantis (1984), (1992) is used. As also used in Altan and Aifantis (1997), Akintayo (2011); Akintayo et al. (2012); Akintayo (2014a, b, c, d), the one-dimensional gradient elasticity stress-strain constitutive equation is taken as

$\sigma_{x}=E\left(\varepsilon_{x}-c \frac{d^{2} \varepsilon_{x}}{d x^{2}}\right)$ 
where $\sigma_{x}$ is the stress and $\varepsilon_{x}=d u / d x$ is the bar strain and $c$ is the gradient elastic coefficient, which is the internal length parameter $l^{2}$ where $l=v \mathrm{c}$. It is obvious that the first part of the stress-strain expression is the classical elastic term, whereas the second part is the newly introduced effect due to higher-order strain gradient which defines strain effect at the scale of the material microstructure.

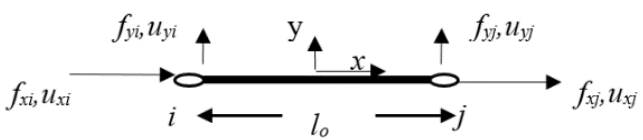

(a)

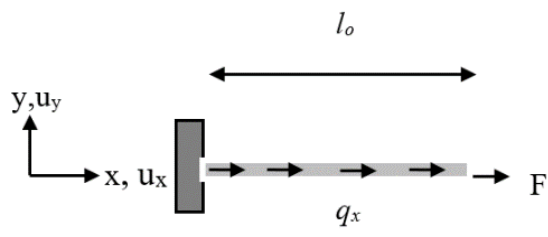

(b)

Figure 3. (a) 2-node truss bar element referred to its local coordinate system $(x, y)$ with nodal forces $f_{i j}$ and displacements $u_{i j}$ (b). A bar under axial tensile load $\mathrm{F}$.

Hence in a similar manner, the gradient elasticity equivalent of the classical equilibrium and governing equation takes the form

$E A\left(\frac{d^{2} u}{d x^{2}}-c \frac{d^{4} u}{d x^{4}}\right)+q_{x}=0$

Similar equations can be obtained using the principle of virtual work Akintayo (2011) and the variational principle Tsepoura et al. (2002). With $q=0$, the solution of Eq. (2) is obtained as

$u=C_{1}+C_{2} x+C_{3}\left(\cosh \frac{x}{\sqrt{c}}+\sinh \frac{x}{\sqrt{c}}\right)+C_{4}\left(\cosh \frac{x}{\sqrt{c}}-\sinh \frac{x}{\sqrt{c}}\right)=0$

where $C_{1}, C_{2}, C_{3}$ and $C_{4}$ are constants to be obtained from the boundary conditions. Akintayo (2014a) presented different combinations of extra non-classical boundary conditions imposed at the support of the bar associated with strain and strain gradient dominated deformation mechanisms, some of which are presented in the next section.

\subsection{Prescribed Non-classical Boundary Conditions and Physical Microstructure Phenomena}

In Akintayo (2011) the natural (or force) boundary condition of the problem were obtained using the principle of virtual work and since higher order boundary conditions are required to solve the governing equation of Eq. 2, Akintayo (2014a) arbitrarily studied several combinations of non-classical higher order boundary conditions imposed at the bar element supports. Moreover, the derivative of displacement (strain) and higher order derivatives of displacements (strain gradients) have been used as extra non-classical boundary conditions in gradient elasticity problems by authors like, Ru and Aifantis (1993), Altan and Aifantis (1997) and Polizzotto (2003).

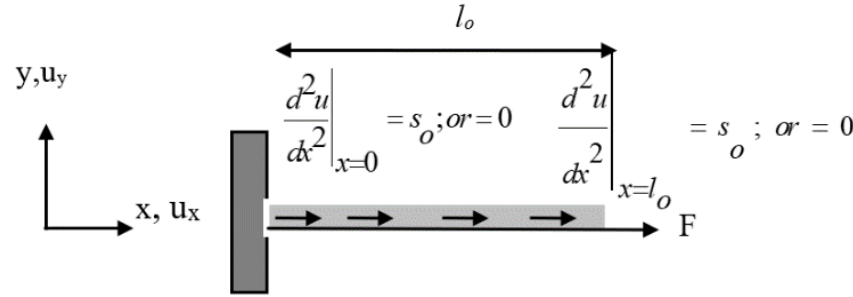

(a)

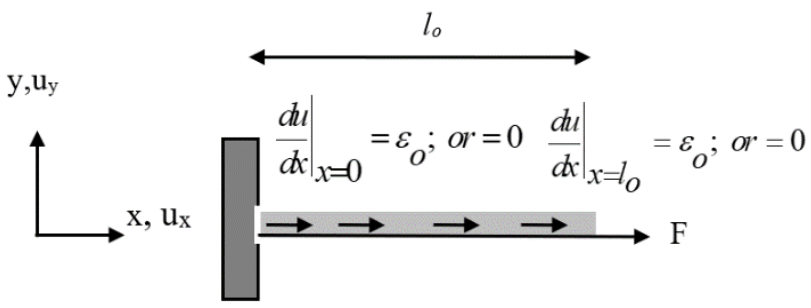

(b)

Figure 4. (a) Strain gradient $\left(s=s_{0}\right)$; $\left(s=-s_{0}\right)$ or $(s=0)$ imposed at either boundary. (b) Strain $\varepsilon=\varepsilon_{0}$ or $\varepsilon=0$ imposed at either boundary.

Sequel to the objective of this paper the boundary conditions are addressed here on the basis of simple physical conditions of the microstructure and at the support in accordance with experiments, not only consistent with the more general mathematical considerations which are based on variational principles or the consideration of additional internal 
lengths. As presented in Akintayo (2014a), at the supports of the undeformed bar, it can be observed that by imposing a zero rate of change of the displacement a stiff condition is imposed at the support so $\varepsilon=0$. On the other hand, by allowing a less stiff condition in which a measure of deformation is prescribed and the rate of change of the displacement is not zero, the imposed strain effect will correspond to the prescribed strain value $\varepsilon=\varepsilon_{0}$. Hence the non-classical boundary condition can be imposed at either end as shown in Figure 4b. A further stiffening or weakening of the support can be achieved by adjusting the rate of change of the strain, thus imposing strain gradients (higher order derivatives of displacement). Consequently, the non-classical boundary condition prescribes strain gradients at the ends of the bar as stiffening or weakening mechanism of the material/bar. It was observed in Akintayo $(2014 a, 2018)$ that bars with imposed strain or imposed strain gradient at the unconstrained boundary were less stiff, while those with imposed strain gradient at the constrained boundary resulted in stiffer bar responses. With a prescribed strain gradient value $s_{0}$ at either end, the boundary conditions are as shown in Figure 4a. Thus, the following six Cases of the combinations of extra non-classical boundary conditions extracted from Akintayo (2014a):

$$
\begin{aligned}
& \left.\frac{d^{2} u}{d x^{2}}\right|_{x=0}=0 \quad ;\left.\quad \frac{d u}{d x}\right|_{x=l o}=0 \\
& \left.\frac{d^{2} u}{d x^{2}}\right|_{x=0}=s_{o} \quad ;\left.\quad \frac{d u}{d x}\right|_{x=l o}=0 \\
& \left.\frac{d u}{d x}\right|_{x=0}=0 \quad ;\left.\quad \frac{d^{2} u}{d x^{2}}\right|_{x=l_{o}}=s_{o}
\end{aligned}
$$

$$
\begin{gathered}
\left.\frac{d^{2} u}{d x^{2}}\right|_{x=0}=0 \quad ;\left.\quad \frac{d u}{d x}\right|_{x=l o}=\varepsilon_{o} \\
\left.\frac{d^{2} u}{d x^{2}}\right|_{x=0}=s_{o} \quad ;\left.\quad \frac{d u}{d x}\right|_{x=l o}=\varepsilon_{o} \\
\left.\frac{d u}{d x}\right|_{x=0}=\varepsilon_{o} \quad ;\left.\quad \frac{d^{2} u}{d x^{2}}\right|_{x=l_{o}}=s_{o}
\end{gathered}
$$

To relate the modelled block in Figure 1 to the bar element of Figures 3 and 4 , the physical implications of the boundary conditions of Akintayo (2014a) are addressed here based on the spatial distribution of inclusions and the spatial variation of strain. Since the physics of the macroscopic phenomena depends only on the principal phenomena on the microscale, the description of the microscopic phenomena should always be greatly simplified and realistic Bazant et al. (1990). Though most materials are an inhomogeneous agglomeration of constituents at the microscale, e.g. particles and matrix material in composites, gravel and cement in concrete, porosity, presence of micro-voids and micro-cracks etc, the material of any block is either homogeneous or heterogeneous. Homogeneous are those with material properties the same at every point and of uniform composition throughout that cannot be mechanically separated into different materials and are invariant upon translation. Heterogeneous are those with material properties that are not the same at every point, but vary upon translation and exhibit dramatic heterogeneity in strength from one domain area to another that can be caused by microstructural heterogeneity, crystal structure heterogeneity or compositional heterogeneity. Thus the domains of the particles, pores, micro-voids and cracks in a matrix will present spatial variation of strain and at this scale the interaction of the various domains will result in underlying phenomenological responses in the neighborhood and in the behavior of the material which can be readily captured.

Higher order deriviates compares the value of a function at a point with the average of the function at neighboring points. In this case, roughly it measures the difference between the local and average values of the displacement in an infinitesimal neighborhood of a point. It can be observed that imposing a zero valued higher order derivative of displacement (a zero-strain gradient effect $s=0$ ) at the point of the node implies that the displacement is at that point equal to the average of its neighbours. Hence at the node of the bar element the average displacement around the neighbourhood will be represented just as is expected for a typically homogeneous matrix and material. On the other hand, imposing or prescribing a non-zero and positive higher order derivative of displacement (a positive strain gradient effect $s=s_{0}$ ) implies that the displacement is smaller at that point than the average of its neighbours. Thus at the node a strengthening effect resulting from a stronger domain will be represented by a stiffer bar. If the imposed higher order derivative of displacement is taken as a negative value strain gradient effect $s=-s_{0}$, it implies that the displacement is higher at that point than the average of its neighbours, resulting in a reverse behavior, hence a weakening effect resulting from a weak domain is represented by a less stiff bar. 
Based on the description given above the response of boundary condition $4^{*}$ and $6^{*}$ below are observed to correspond to the boundary conditions 4 and 6 . Hence for consistency the boundary conditions 4 and 6 are replaced here by the following:

(4*) $\left.\frac{d^{2} u}{d x^{2}}\right|_{x=0}=-s_{o} \quad ;\left.\quad \frac{d u}{d x}\right|_{x=l o}=0$

$$
\left.\frac{d^{2} u}{d x^{2}}\right|_{x=0}=-s_{o} \quad ;\left.\quad \frac{d u}{d x}\right|_{x=l o}=\varepsilon_{o}
$$

These six boundary conditions result in different displacement values at the bar node. From observation the absence of the prescribed strain (i.e. when $\varepsilon=0$ ) will result in the coupling of the following cases: Case 2 reduces to Case 1 ; Case 5 to Case 3; and Case 6 to Case 4 respectively. However, to qualitatively quantify the assumed imposed strain gradient value $s_{o}$ it becomes necessary to qualitatively relate it to some physical parameter. This is addressed in Chapter 3 .

\subsection{Stiffness and Displacement-Force Relations for the GTM Bar Elements}

Like in the classical case the bar stiffness is obtained using the unit displacement method with the displacement at the free end $x=I_{0}$ of the bar of Figure 3 evaluated from the force expression. The displacement is obtained from Eq. 3 using the different boundary conditions of Eq. 4 and 5. In Akintayo (2018) a generic stiffness expression was derived to effectively capture the various mechanisms represented by the six cases in Eq. 4 and 5, which is given as:

$k_{\text {generic }}^{g}=\frac{E A\left(1-\varepsilon_{o} \sqrt{c} \tan h \frac{l_{o}}{\sqrt{c}} \pm c s_{o}\left(1-\sec h \frac{l_{o}}{\sqrt{c}}\right)\right.}{\left(l_{o}-\sqrt{c} \tan h \frac{l_{o}}{\sqrt{c}}\right)}$

From Eq. 6 the six cases can be obtained respectively as follows:

Case 1 is obtained by setting $\varepsilon_{0}=0$ and $s_{0}=0$ (hence second and third terms vanish and this represents a virgin material behaviour equal to the average of its neighbours and without initial strain $\varepsilon_{0}$ );

Case 2 is obtained by setting $\varepsilon_{0}>0$ and $s_{0}=0$ (hence third term vanish and this represents a material behaviour equal to the average of its neighbours but with initial prescribed strain $\left.\varepsilon_{0}\right)$;

Case 3 is obtained by setting $\varepsilon_{0}=0$ and $+s_{0}$ third term retained (hence second term vanish and this represents a material behaviour stiffer at that point than the average of its neighbours due to strengthening strain gradient effect);

Case 4 or $4^{*}$ is obtained by setting $\varepsilon_{0}=0$ and $-s_{0}$ third term retained (hence second term vanish and this represents material behaviour less stiff at that point than the average of its neighbours due to a weakening strain gradient effect); Case 5 is obtained by setting $\varepsilon_{0}>0$ and $+s_{0}$ third term retained (hence this represents a material behaviour stiffer at that point than the average of its neighbours due to strengthening strain gradient effect and prescribed strain $\varepsilon_{0}$ );

Case 6 or $6^{*}$ is obtained by setting $\varepsilon_{0}>0$ and $-s_{0}$ third term retained (hence this represents material behaviour less stiff at that point than the average of its neighbours due to a weakening strain gradient effect and prescribed strain).

A generic displacement-force relation can be obtained as

$u_{\text {generic }}^{g}=\frac{F\left(l_{o}-\sqrt{c} \tan h\left(\frac{l_{o}}{\sqrt{c}}\right)\right)}{E A}+\varepsilon_{o} \sqrt{c} \tan h\left(\frac{l_{o}}{\sqrt{c}}\right) \pm c s_{o}\left(1-\sec h \frac{l_{o}}{\sqrt{c}}\right)$

From Eq. 7 the displacement for the six cases can be obtained respectively as follows: Case 1 is obtained by setting $\varepsilon_{0}$ and $s_{0}$ to zero (hence second and third terms vanish); Case 2 is obtained by setting so to zero (hence third term vanish); Case 3 is obtained by setting $\varepsilon_{0}$ to zero (hence second term vanish) and the negative sign of term three retained; Case 4 or $4^{*}$ is obtained by setting $\varepsilon_{0}$ to zero (hence second term vanish) and the positive sign of term three retained; Case 5 is obtained using the complete expression and the negative sign before the third term retained; Case 6 or $6 *$ is obtained using the complete expression and the positive sign before the third term retained. From observation, the stiffness and displacement expressions of Eq. 6 and 7 reduce to the classical case in the absence of the gradient terms. 


\section{QUALITATIVE REPRESENTATIONS OF THE CHARACTERISTIC INTERNAL LENGTH AND STRAIN GRADIENT IN GTM}

\subsection{The Charateristic internal length of Homogeneous and Heterogeneous Materials for GTM}

As indicated in Chapter 2 the characteristic internal length is the parameter that scales the strain gradient effect. Yet its determination is by no means trivial and it is usually approached from the consideration of the internal material structure at the microscopic level and then fitting it to the available results of the macroscopic experiments. The noteworthy observations made in Chapter 2 suggest that by a careful consideration of existing principal microstructure material parameters it is possible to describe the principal microscale phenomena. Thus based on a statistical deterministic approach qualitatively obtain a value range for the characteristic internal length for use in the GTM. Though such detailed methodology is beyond the scope of this paper, a simple illustration is briefly presented here.

The two generalised classifications of materials are considered: homogeneous and heterogeneous materials. The domain sizes could be in the range of micrometers to millimeters and the domain geometry can vary to form very diverse material systems. At the microscale of the homogeneous material like copper, the grain is usually the controlling microstructure parameter and the surface may not reveal too large a difference in grain size. Figure 5 shows the schematic of the real grain structure and the homogeneous equivalent of polycrystalline wires Xi et al. (2011).

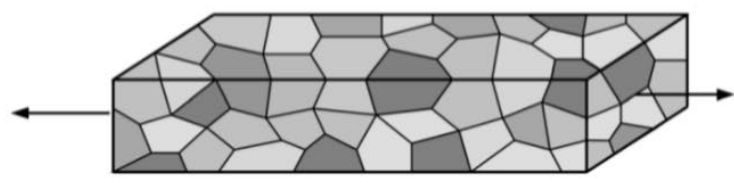

(a)

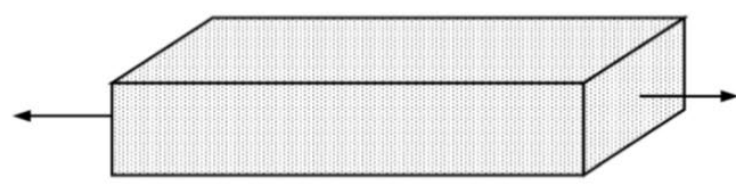

(b)

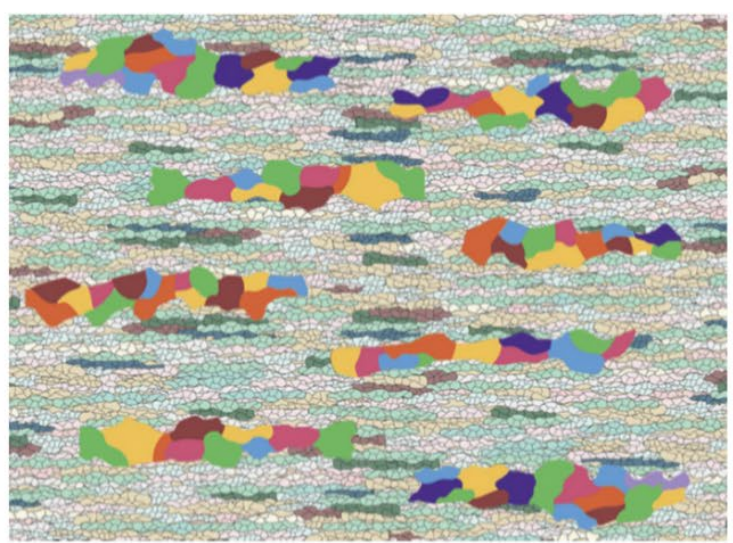

(c)

Figure 5. Schematic of polycrystalline wires in: (a) real grain structure; (b) equivalently homogeneous structure (obtained from $X i$ et al. (2011)); c Schematics of heterogeneous lamella structure with elongated soft coarse-grained domains embedded in an ultrafine-grained matrix (obtained from Wu et al. (2015)).

The characteristic dimension of the polycrystalline wire is the average grain size while the length of the wire is usually a macroscale dimension. Thus, the characteristic internal length $l$ can be determined based on the length scale of the grain. Hence the lower values of $\ell$ observed for copper as compared to other materials in Table 1.

On the other hand, for heterogeneous materials like the lamella structure with elongated soft coarse-grained domains embedded in an ultrafine-grained matrix, shown in Figure 5, there are huge microstructural heterogeneities in these materials Wu et al. (2015). The same occurs for fibre reinforced composite materials. In Geers et al. (1999) several aspects regarding the nonlocal character of the damage process for short glass fibre-reinforced polypropylene (SGFPP) were addressed and the characteristic internal length was determined using strain fields from an experimental analysis. The glass fibre content of the SGFPP plates was $30 \mathrm{wt} \%$ with an average fibre length of approximately $12.5 \mathrm{~mm}$ randomly distributed in the matrix and a value of $c$ between the lower and upper range of $1 \mathrm{~mm}^{2}$ and $5 \mathrm{~mm}^{2}$ was observed (where $\ell=\mathrm{V} c=1 \mathrm{~mm}-2.236 \mathrm{~mm}$ ). Although the particle size of polypropylene could range between 0.03 to $1.0 \mu \mathrm{m}$, Paik and Kar (2009), Lotti et al (2000), the characteristic internal length obtained depended on the composite nature of SGFPP and the heterogeneity of the micro and meso scales. It can be observed that the characteristic internal length is in the millimeter scale which is the length scale of the reinforced fibre length $(12.5 \mathrm{~mm})$. Moreover, in composite materials the maximum size of heterogeneity is controlled by the largest particles and thus this will influence the scale of the characteristic internal length Ben Amoz (1976). Hence it can be observed that these simple parameters, the matrix particle size, length and cross-section thickness of the reinforcement fibres/particles and spacing within the material are major factors that influence the characteristic internal length of the heterogeneous material.

The spacing between particles is an important geometrical parameter for controlling an inhomogeneous plastic deformation in the composite Kamat et al. 1991. Generally, in such materials this dimension could be the average 
distance between the fibers/particles and it is in fact an idealized description of a statistical distribution inside it. For instance, the growth of microcracks is at a scale smaller than the particle/fibre sizes but usually in the scale of the grains of the matrix. Hence the characteristic internal length could be determined as the scale of a unit representative volume of the material where damage will localize. Moreover, it has been observed that at a fixed volume fraction of the particles, the smaller the inclusion particle size is the smaller is the particle spacing and the higher the strain gradient Dai et al. 2004. Consequently, in Figure 6 it is assumed that the dimension of a unit volume consisting of both the matrix and the inclusion will depend on their volume fractions.

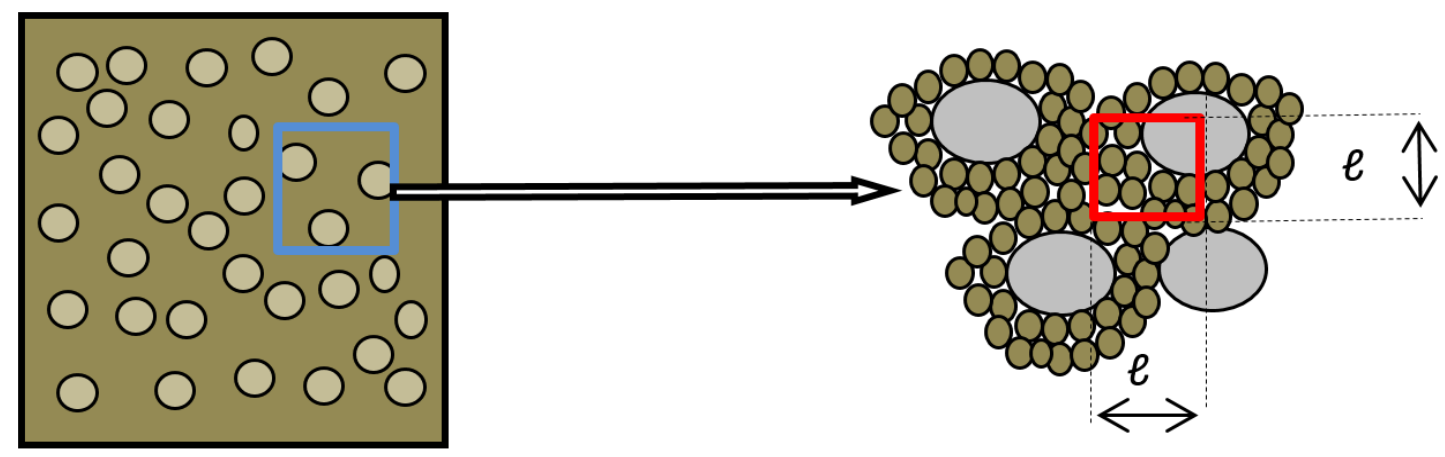

(a)

(b)

Figure 6. Schematic of microstructure of composite material: (a) microstructure of a representative volume of a composite material; (b) Composite unit volume in red with dimension based on matrix and inclusion volume fraction.

For simplicity details of the crystallographic and morphologic orientations, shapes of the grains and particles and interfaces are neglected. Hence in the simplest form based on the rule of mixtures the microstructure dimension of the unit cell defines the characteristic internal length $\ell$ as,

$\ell=\left(V_{m} d_{m}\right)+\left(V_{i} d_{i}\right)$

Where $V_{m}$ and $V_{i}$ are the volume fractions of the matrix and inclusion (particle or fibre) respectively, while $d_{m}$ and $d_{i}$ are the largest matrix and inclusion particle/grain sizes respectively. Hence based on Eq. 8, and the aforementioned properties of SGFPP the value of $\ell$ is obtained as $\approx 3.83 \mathrm{~mm}$ comparable to $1 \mathrm{~mm} \leq \ell \leq 2.236 \mathrm{~mm}$ obtained in Geers et al. (1999). Moreover to obtain a lower range, a value half that obtained is taken since stress is built progressively from the fiber ends up to a certain length (fiber critical size) where the stress transferred to the fibers reaches the characteristic maximum value for a specific system Mallick (1997). Thus the predicted range is taken between $1.9 \mathrm{~mm} \leq \ell \leq 3.8 \mathrm{~mm}$.

This is again illustrated using the porous metal fiber/powder sintered composite sheet (PMFPSCS) studied in Zou et al. (2016). It was developed by sintering a mixture of a porous metal fiber sintered sheet (PMFSS) and copper powders with particles of spherical shape. The copper particles were found to be between the range of $25-100 \mu \mathrm{m}$ while the copper fiber ( $80 \%$ porosity) produced with an equivalent diameter of $100 \mu \mathrm{m}$ was segmented into short fibers of $15 \mathrm{~mm}$ length, but the characteristic internal length was not obtained. From the aforementioned as observed for SGFPP and based on the dimensions of the copper particles and the copper fibres in PMFPSCS the scale of the characteristic internal length can be envisaged to also fall within the millimeter length scale. However the porosity of the fiber will have a significant effect. Based on Eq. 8 and the aforementioned dimensions of the matrix and inclusions, the characteristic internal length for the PMFPSCS material is obtained as $3.08 \mathrm{~mm}$. Hence in the proceeding sections the predicted range of $1.5 \mathrm{~mm} \leq \ell \leq 3 \mathrm{~mm}$ is adopted and based on this the behavior of the material will be qualitatively verified and compared to the deformation observed in the experiment of Zou et al. (2016).

\subsection{Relation between the Imposed Strain Gradient, Characteristic Internal Length and Deformation Parameter}

In the gradient elastic analysis of the tensile loaded bar of Fig. 3, the strain gradient effect is considered and the displacement u Eq. 7 includes the effect of the microstructure deformation. Usually, the deformation mechanism at the microscale is dislocation motion and production within the grains of the material, while grain boundaries act as obstacles creating a strengthening boundary zone or sources of dislocation motion or production. Effectively, this mechanism can be shown to be responsible for the development of higher order strain or stress gradients at the macroscale and the 
transportation of strain from one material region to another Aifantis (1994). It is well established that dislocation-based mechanisms can be satisfactorily used at the macro, micro and at the nano-scales in describing strain gradient influence in material behaviour Fleck et al (1994) and Gao et al (1999). Therefore intuitively, the strain gradient effect will be present as long as crystal grains contain dislocations or there exist a spatial variation of strain. The spatial variation of strain may be due to initial inhomogeneity of the microstructure or heterogeneity developed during deformation.

In this paper it is assumed that as long as there exists a spatial variation of strain in the material, the nature of the underlying phenomena can be captured through the imposed strain gradient according to the conditions of Section 2.4: $\left(s_{0}=0, s_{0}>0\right.$ or $\left.s_{0}<0\right)$ and qualitatively scaled with the characteristic internal length. Hence to achieve this in a simply manner, the deterministic problem then resides in deriving a relation to qualitatively quantify the imposed strain gradient $\mathrm{s}_{\mathrm{o}}$. The simple approach adopted here is to relate the characteristic internal length and the strain gradient to measurable deformation parameters. It has been observed that variation in strain gradient in experiments may result in significant deviation in the mechanical properties and a non-uniform test is required that triggers inhomogeneous deformations and the need for accurate local measurements to be made in the localization zone Geers et al. (1999).

Moreover, Akintayo (2014b) observed that the cross-sectional area change in a bar during deformation and the elastic modulus Akintayo (2014c) depend on the size of the microstructure: the smaller the size of the characteristic internal length the smaller was the cross-sectional area change and higher the elastic modulus. Hence it is assumed here that since the characteristic internal length scales the strain gradient effect any measurable failure or deformation parameter $\lambda_{i}$ will depend on $\ell$ as the length of the representative material volume where damage localizes Sun and Honig (1994). Accurate local measurements can be made in any such localization zone Geers et al. (1999). Materials with small values of characteristic internal length will correspond to lower values of the cross-sectional area changes and consequently the localized neck and the deformation parameter. Hence in the simplest form the maximum measure of the deformation parameter at failure $\lambda_{f}$ can be defined in terms of $\ell$ as:

$\lambda_{f}=(\beta \ell)$

where $\beta$ is taken as a ratio between the measure of localization or deformation zone $\lambda_{f}$ and the characteristic internal length $\ell=\sqrt{ } c$ which varies during deformation. To prevent the damage zone from localizing to a zero volume the ratio $\beta$ is taken to be greater than zero $\beta=1$ Bazant and Pijaudier-Cabot (1989). Hence the parameter $\lambda_{i}$ is a general representative characteristic deformation parameter. According to the study of Dai et al. 2001 and Lui et al. 2003 inhomogeneous plastic deformation can be effectively characterized by the strain gradient. In addition, an increase in the strengthening boundary zone corresponds to an increase in the strain gradient, hence as the bar deforms the width of the deformation parameter $\lambda_{i}$ will depend on the resistance from the strain gradient $s_{i}$ within the localization or deformation zone: as $s_{i}$ decreases, $\lambda_{i}$ will increase. This in its simplest form can be qualitatively expressed as,

$\lambda_{f}=\left(s_{f}\right)^{-1}=(\beta \ell)^{-1}$

By substituting the expression for $\lambda_{f}$ given in Eq. 9 into Eq. 10 a simple relation is established between the strain gradient during deformation $s_{i}$ and at failure $s_{f}$ and the characteristic internal length $\ell=\mathrm{V} c$, such that as the latter decreases the former increases., The strain gradient $s_{f}$ at failure becomes:

$s_{f}=\left(\lambda_{f}\right)^{-1}=(\beta \ell)^{-1}=\frac{1}{\beta \sqrt{c}}$

Hence with this the characteristic internal length remains the constitutive length parameter that scales the gradient effect Abu Al-Rub and Voyiadjis (2004). This can also be related to the total dislocation density, the density of the Geometrically Necessary Dislocation GND $\rho_{G}$ and Statistically Stored Dislocation SSD $\rho_{s}$ in a simple manner Fleck et al (1994). However, this is beyond the scope of this paper. Consequently, for the GTM bar element the imposed strain gradient $s_{o}$ can be readily determined based on Eq. 11. Moreover strain gradient dominated deformation experiments at the micro-scale have also shown remarkable strengthening effect with a proposed strain gradient in the order of the inverse of a characteristic length scale Fleck et al. (1994) and Gao et al. (1999).

With the simple relation of Eq. 11 and by considering the characteristic internal length of any material from existing experiments or a statistical deterministic evaluation, the strain gradient condition $s_{o}$ imposed at the gradient bar element 
support can be readily obtained for different deformation stages. The behaviour of the different metals of Table 1 is revealed in the graphs of Figure 7 for the variation of strain gradient $s_{o}$ as the ratio $\beta$ changes.
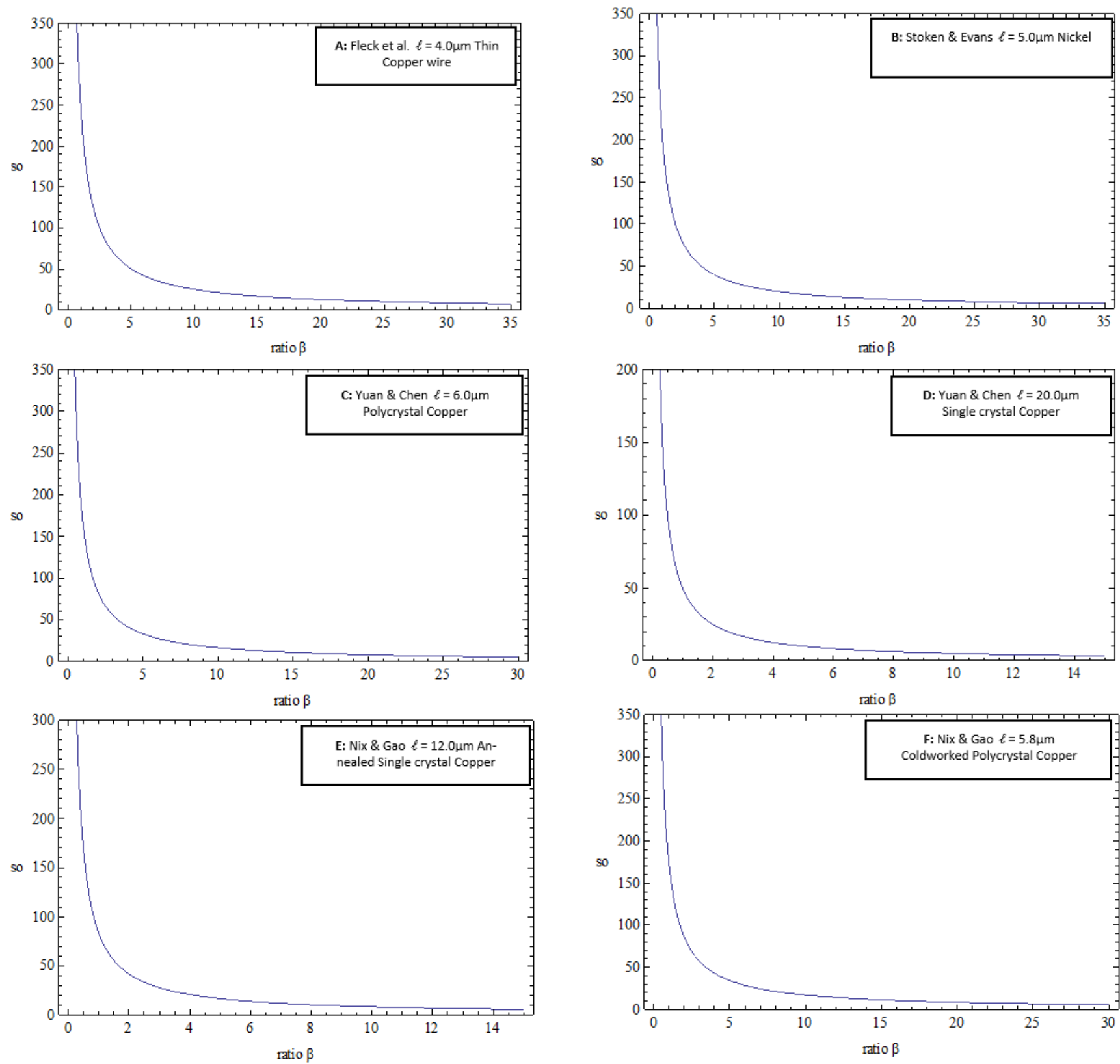

Figure 7. (a) Strain gradient $s_{0}$ plotted against $\beta$ the ratio between the deformation parameter $\lambda_{f}$ and the characteristic internal length $\ell<20 \mu \mathrm{m}$ for various materials in Table 1.

As expected, the strain gradient values are shown to be higher for materials with smaller characteristic internal length. For instance based on the characteristic internal lengths of the different copper materials of Table 1, in Figure 7 the corresponding strain gradient values $s_{o}$ at $\beta=1$ are the following: (M1: $\left.\ell=4 \mu \mathrm{m}, s_{o}=250\right),\left(\mathrm{M} 6: \ell=5.84 \mu \mathrm{m}, s_{o}=171.2\right)$, (M3: $\left.\ell=6 \mu \mathrm{m}, s_{0}=166.7\right)$, (M5: $\left.l=12 \mu \mathrm{m}, s_{0}=83.3\right)$, (M4: $\left.l=20 \mu \mathrm{m}, \mathrm{s}_{0}=50\right)$. As the materials undergo deformation $\beta$ increases and they exhibit a significant $50 \%$ drop in the strain gradient value between the ratio $1<\beta \leq 2$, with $s_{o}$ at $\beta=2$ obtained as: (M1: $\left.\ell=4 \mu \mathrm{m}, s_{0}=125\right)$, (M6: $\left.\ell=5.84 \mu \mathrm{m}, s_{0}=85.6\right)$, (M3: $\left.\ell=6 \mu \mathrm{m}, s_{o}=83\right)$, (M5: $\left.\ell=12 \mu \mathrm{m}, s_{0}=41.7\right)$, (M4: $\left.\ell=20 \mu \mathrm{m}, s_{o}=25\right)$. Thereafter, another $20 \%$ between $2<\beta \leq 10$ with $s_{o}$ values (M1: $\left.\ell=4 \mu \mathrm{m}, s_{o}=25\right),(\mathrm{M} 6: \ell=5.84 \mu \mathrm{m}$, $\left.s_{o}=17.12\right),\left(\mathrm{M} 3: \ell=6 \mu \mathrm{m}, s_{o}=16.7\right),\left(\mathrm{M} 5: \ell=12 \mu \mathrm{m}, s_{o}=8.33\right),\left(\mathrm{M} 4: \ell=20 \mu \mathrm{m}, s_{o}=5\right)$. For the regime $10 \leq \beta<15$ the remaining $30 \%$ of the resisting effect is depleted until the material completely fails (M1: $\ell=4 \mu \mathrm{m}, s_{0}=16.7$ ), (M6: $\left.\ell=5.84 \mu \mathrm{m}, s_{o}=11.4\right)$, (M3: $\left.\ell=6 \mu \mathrm{m}, s_{o}=11.11\right)$, (M5: $\left.\ell=12 \mu \mathrm{m}, s_{o}=5.6\right),\left(\mathrm{M} 4: l=20 \mu \mathrm{m}, s_{o}=3.33\right)$. This is suggestive that the deformation resisting effect induced by strain gradient is highest between $1<\beta<2$ where it corresponds to the 
effect of prior dislocation density, which can be a combination of the density of the SSDs and that of the GNDs that is a function of the strains nucleated during the specimen preparation Alsaleh (2004). Gao et al. (1999) observed that the strain gradient effects was more significant when the density of the SSDs and GNDs were of the same order of magnitude and the effective strain gradient was taken to be proportional to the deformation true strain and inversely proportional to the characteristic length of the microstructure.

For $\beta>1$ it can be deduced that the material yielding point is reached and subsequently the plastic deformation and strain hardening regime takes place between $1<\beta \leq 10$, with continuous changes in the densities of the GND and SSD during the process and the GNDs taking preeminence in the strengthening due to strain gradient. In the plastic region, the dislocation in the material increases and the dislocation interaction is repulsive in nature and as the dislocation density increases the further deformation of the material becomes difficult, resulting in work hardening or strain hardening. During the hardening regime, granular materials behave as a continuum until the failure or the instability point where deformations begin to localize into a small but finite shear zone. The material stops behaving as one block and is divided into several independent fragments that split by the shear bands failure zones Voyiadjis and Song (2006). The regime between $10 \leq \beta<15$ may be associated with the remaining resistance of the material as it approaches complete failure. Figure 8 shows the engineering stress-strain curve for copper with an enlarged scale, indicating strains from zero up to specimen fracture; it shows that the rate of strain hardening diminishes up to a point labeled UTS (Ultimate Tensile Strength). Beyond that point, the material appears to strain soften, so that each increment of additional strain requires a smaller stress which is similar to the behavior observed within the regime $(10 \leq \beta<30)$, $\beta$ value increases more rapidly than the decrease in the strain gradient. An additional observation is the much more prolonged extension of the process at $\beta>30$ for the following materials: (M1: $\ell=4 \mu \mathrm{m}, s_{o}=8.33$ ) and (M6: $\ell=5.84 \mu \mathrm{m}, s_{o}=6.7$ ): which confirms the effect of higher strain gradient presence for materials with smaller sized characteristic internal length.

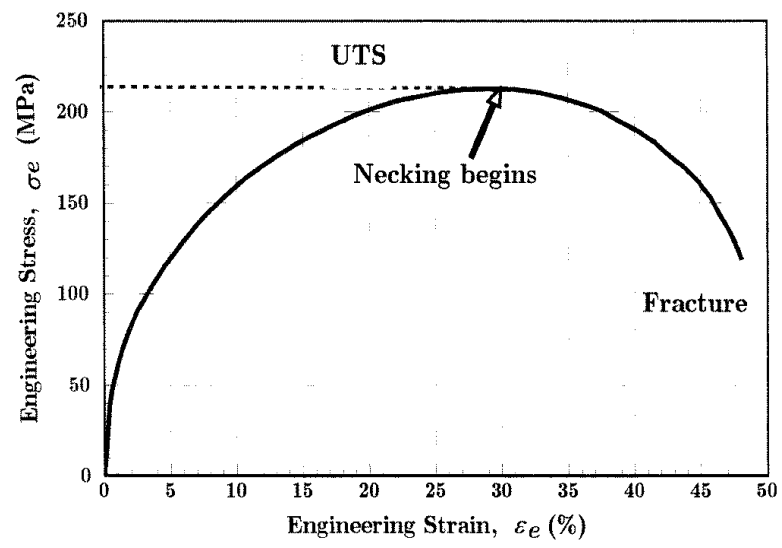

Figure 8: Full engineering stress-strain curve for annealed polycrystalline copper (Roylance (2001)).

On the other hand, in Figure 9 the other materials exhibit much lower strain gradient values at $\beta=1$ : (M7: $\left.\ell=0.33 \mathrm{~mm}, s_{o}=3\right)$, (M8: $\left.\ell=0.62 \mathrm{~mm}, s_{0}=1.61\right)$, (M10: $\left.\ell=0.77 \mathrm{~mm}, s_{o}=1.3\right),\left(\mathrm{M} 9: \ell=0.80 \mathrm{~mm}, s_{o}=1.25\right.$, (M11: $\left.\ell=2 \mathrm{~mm}, s_{o}=0.5\right)$, (M12: $\left.\ell=2.236 \mathrm{~mm}, s_{o}=0.45\right)$. These are close to the values obtained for the previous set of materials for $\beta$ values between $15<\beta \leq 30$, thus indicating these materials will exhibit lower resistance to deformation. As expected the larger values of their characteristic internal length is responsible for these lower values. Subsequently at $\beta=2$ the following are obtained respectively: (M7: $\left.\ell=0.33 \mathrm{~mm}, s_{o}=1.5\right),\left(\mathrm{M} 8: \ell=0.62 \mathrm{~mm}, s_{o}=0.805\right),(\mathrm{M} 10: \ell=0.77 \mathrm{~mm}$, $\left.s_{o}=0.65\right)$, (M9: $\ell=0.80 \mathrm{~mm}, s_{0}=0.625$, (M11: $\ell=2 \mathrm{~mm}, s_{0}=0.25$ ) (M12: $\ell=2.236 \mathrm{~mm}, s_{0}=0.225$ ), with a $50 \%$ drop. Furthermore at $\beta=4$ the $s_{o}$ values is as low as: (M7: $\left.\ell=0.33 \mathrm{~mm}, s_{0}=0.774\right),\left(\mathrm{M} 8: \ell=0.62 \mathrm{~mm}, s_{0}=0.402\right)$, (M10: $\left.\ell=0.77 \mathrm{~mm}, s_{o}=0.325\right)$, (M9: $\ell=0.80 \mathrm{~mm}, s_{0}=0.151$, (M11: $\left.\ell=2 \mathrm{~mm}, s_{0}=0.125\right),\left(\mathrm{M} 12: \ell=2.236 \mathrm{~mm}, s_{o}=0.113\right)$.

Invariably, for the nonlocal character of the failure behavior of the short glass fibre-reinforced polypropylene SGFPP of graph F in Fig. 9 it can be qualitatively identified that between $0<\beta \leq 2$ about $50 \%$ of the initial resisting effect of strain gradient is depleted. This may be associated with the matrix deformation and matrix cracking observed in the deformation process before the Fibre-matrix debonding. Thereafter, a further sharp drop of $45 \%$ between $2<\beta \leq 4$ until probably the fiber pull out and then complete failure between $4 \leq \beta<10$ and consequently the depleting of the remaining $5 \%$. This is verified from the observation of Geers et al. (1999) which indicated that the maximum distance of localization or deformation zone was between $5 \leq \beta<10$ and the Fibre-matrix debonding and fiber pull-out were characterized by significant displacements and high strains. 

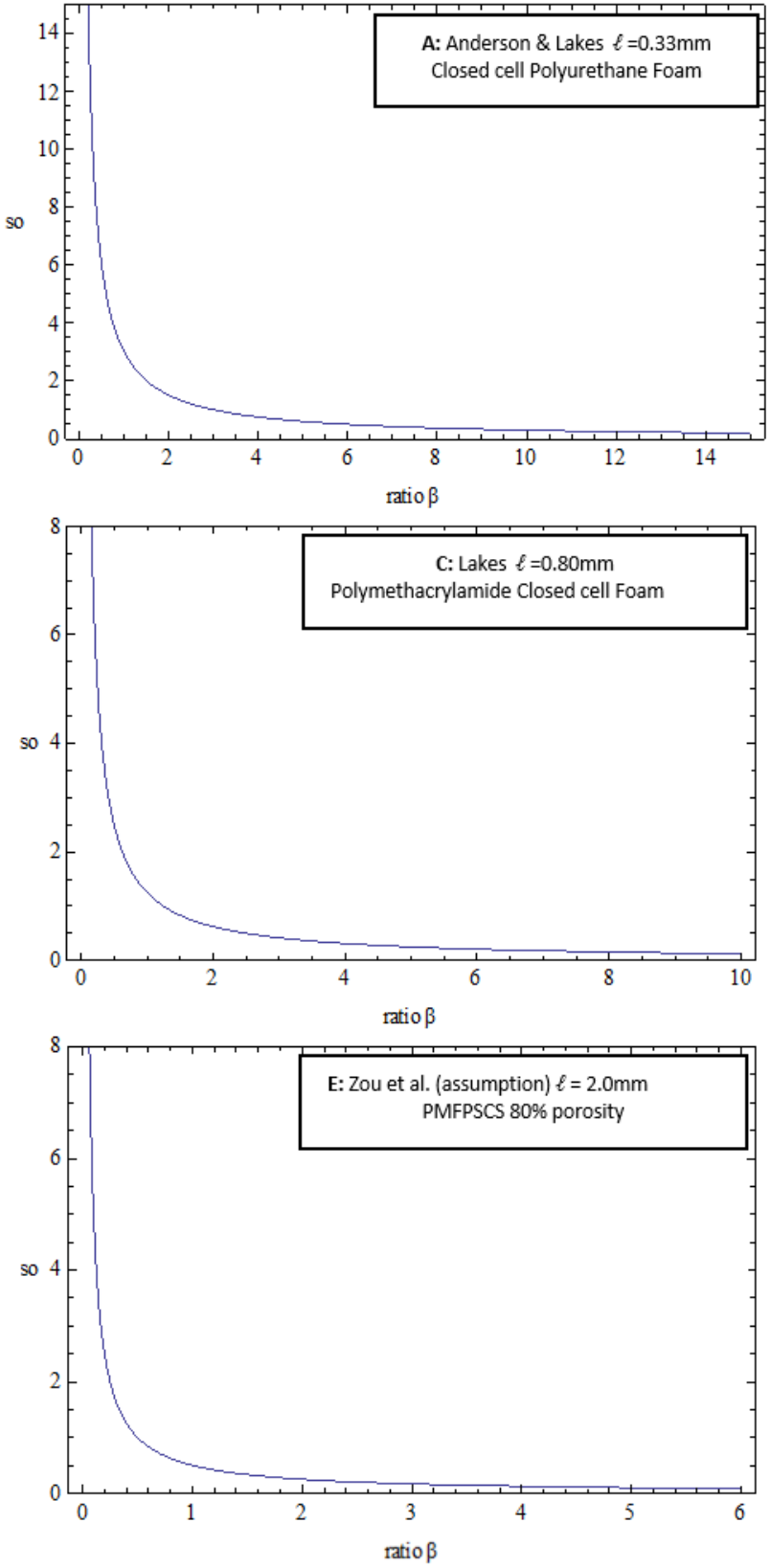
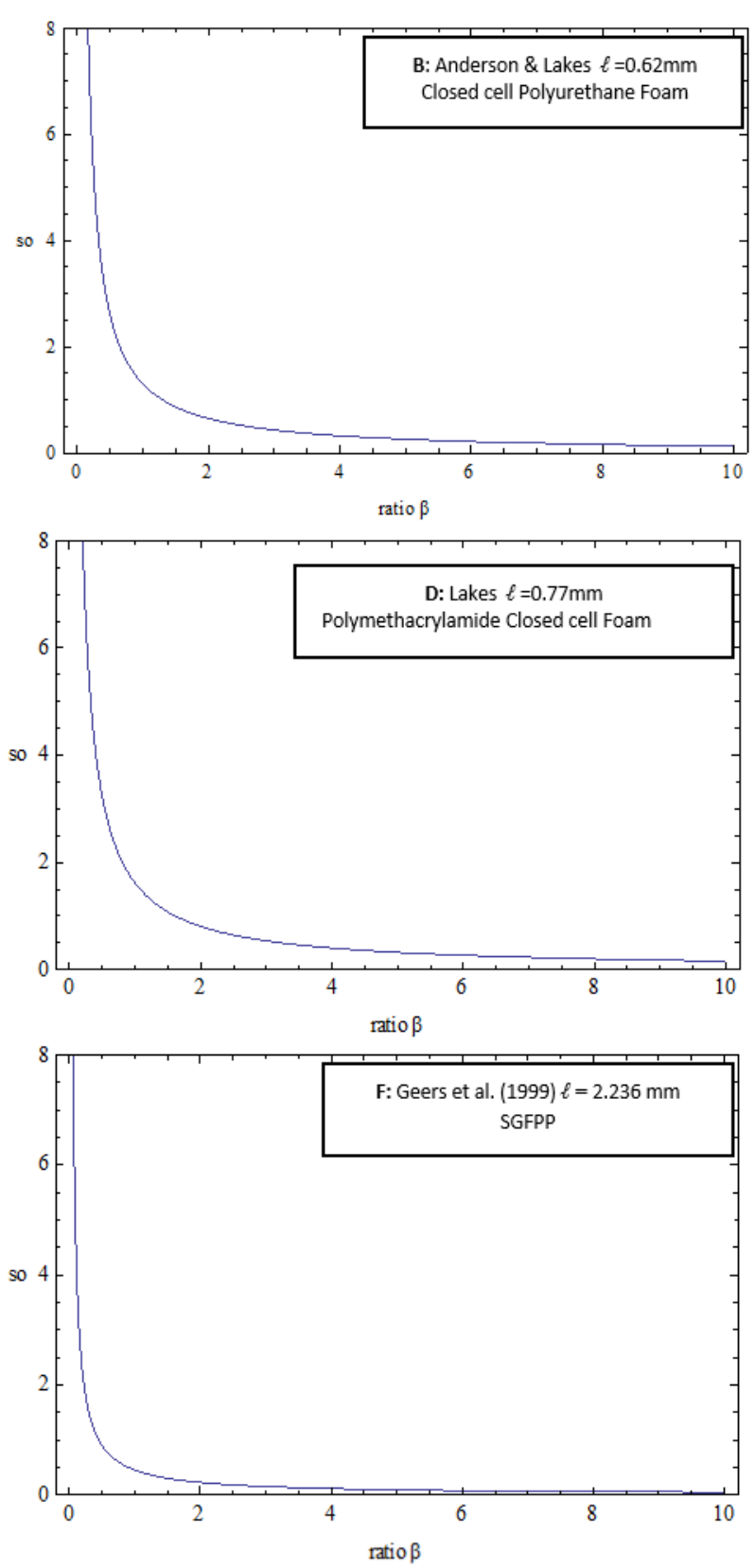

Figure 9. Strain gradient $s_{o}$ plotted against $\beta$ the ratio between the deformation parameter $\lambda_{f}$ and the characteristic internal length $\ell>250 \mu \mathrm{m}$.

For Case E of Fig. 9 with the assumed characteristic internal length of $2 \mathrm{~mm}$ taken for the PMFPSCS, the strain gradient strengthening effect has a less drastic drop at $\beta=4,\left(\mathrm{M} 11: \ell=2 \mathrm{~mm}, s_{0}=0.125\right)$ compared to Case $\mathrm{F}$ which suggests a higher resistance to deformation. Zou et al. (2016) observed that at the initial elastic stage the structural elastic deformation was dominant, and at the hardening stage, the plastic deformation was composed of the structural deformation and the copper fibres' plastic deformation. After the stress reached the maximum value, the PMFPSCS sample began to fracture. Compared with other stages, the elastic stage was very short and the strain was only about $0.05 \%-0.08 \%$. The reason for their observation can be readily seen from graph $\mathrm{E}$ of Figure 9 . The resistance to deformation due to the strengthening effect of strain gradient and the extent of plastic flow are short spanned as indicated by the percentage reduction of strain gradient compared to other materials at the same value of $\beta$.

Although the choice of the assumed value $\ell=2 \mathrm{~mm}$ was based on relatively simple statistical deterministic evaluation, it can be seen that the extent of changes in the strain gradient effect was captured and the material behavior is shown to qualitatively relate to the deformation process observed in the experiment of Zou et al. (2016). In Figure 10 the different characteristic internal length $\ell^{2}=1.0,2.0,3.0,4.0 \mathrm{~mm}^{2}$ assumed for PMFPSCS show that the higher the 
value of the characteristic internal length $\ell$ the lower the strain gradient effect. With these modest observations it is suggestive that a more detailed statistical deterministic approach to arrive at a qualitative characteristic internal length value for modelling with the GTM bar elements may be explored. Moreover, an important factor to be considered in modeling with the GTM bar element is that by assuming a range of values for the determined characteristic internal length the rate of accuracy in capturing the material behavior is increased.

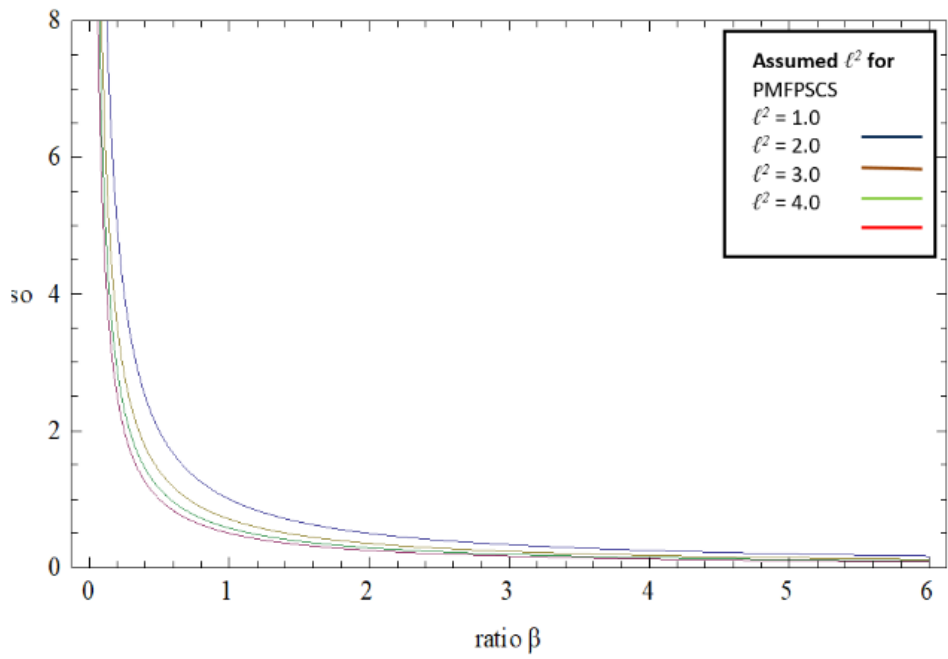

Figure 10. Strain gradient $s_{\circ}$ plotted against $\beta$ the ratio between the deformation parameter $\lambda_{f}$ and the characteristic internal length $\ell^{2}=1.0,2.0,3.0,4.0 \mathrm{~mm}^{2}$ for PMFPSCS.

\section{MODELLING WITH THE GTM BAR ELEMENTS (GTM BE)}

\subsection{GTM Bar Elements Types and their Microstructure Representation}

In the classical elasticity formulation, the stiffness $k$ measures the amount of deflection that the load causes in the material and it is a structural property influenced by the geometry of the specimens as well as the intrinsic material property. On the other hand, for the gradient enhanced case the effect of the microstructure is captured in the stiffness expression $k$ of Eq. 6. Based on the six cases and their coupling earlier identified in Section 2.4 and from the stiffness equation Eqs. 6, the corresponding stiffness values for the copper materials of Table 1 are herein examined: (M1: $\ell=4 \mu \mathrm{m}),(\mathrm{M} 6: \ell=5.84 \mu \mathrm{m}),(\mathrm{M} 3: \ell=6 \mu \mathrm{m}),(\mathrm{M} 5: \ell=12 \mu \mathrm{m})$ and (M4: $\ell=20 \mu \mathrm{m})$.

As shown in Table 2 to reveal the capacity of the GTM bars to capture the microscale effect a large ratio $\gamma=I_{0} / \ell>1000$ is examined between the bar element length and the characteristic internal length. The classical elastic bar element stiffness $k^{c}=44400$ is constant, while the GTM cases account for the copper materials based on their different characteristic internal length regardless of the large ratio $\gamma$. In the absence of the imposed (prescribed) strain $\varepsilon_{0}=0$ and strain gradient $\mathrm{S}_{\mathrm{o}}=0$, Eq. 6 reduces to $\mathrm{k}^{91}$ Case 1 , a GTM bar response that only captures the deformation at the microstructure of a material based on the average condition around the neighbourhood. Hence the following values: (M1: $\left.\ell=4 \mu \mathrm{m}, k^{g 1}=44417.8\right),\left(\mathrm{M} 6: l=5.84 \mu \mathrm{m}, k^{91}=44425.9\right),\left(\mathrm{M} 3: l=6 \mu \mathrm{m}, k^{g 1}=44426.7\right),\left(\mathrm{M} 5: l=12 \mu \mathrm{m}, k^{g 1}=44453.3\right)$ and (M4: $\ell=20 \mu \mathrm{m}, 44453.3$ ). On the other hand, Case 2 GTM bar with stiffness represented as $k^{92}$ includes the effect of the prescribed strains $\varepsilon_{0}$. As illustration to capture the strain effect, the comparison of the stiffness of Case $1 k^{g 1}$ and Case $2 k^{92}$ for prescribed $\varepsilon_{0}$ values $0.0,0,005,0.01,0.05,1.1$ and 2.0 shows the stiffness values of Case 2 , $\left(k^{g 2}\right)$ to decrease as the prescribed strain $\varepsilon_{0}$ increases. This indicates that Case 2 bar models any condition different from the unstrained state having a pre-existing condition within the material represented as prescribed strain which can be specified for each truss element as a "predefined condition". Hence Cases 1 and 2 form the first coupled cases.

Furthermore in addition to the initial prescribed strain $\varepsilon_{0}$, the stiffness values of Table 3 show the effect of a positive or negative prescribed strain gradient $s_{0}$ is also captured using Cases $5\left(k^{95}\right)$ and $6\left(k^{g 6}\right)$ respectively. Case $3\left(k^{93}\right)$ only captures the effect of a positive prescribed strain gradient $s_{o}$ and Case $4\left(k^{g 4}\right)$ the effect of a negative prescribed strain gradients $-s_{o}$. The effect of the prescribed strain on the material under positive strain gradient $s_{o}$ is readily seen as the prescribed strain increases so does Case 5 get less stiff than Case 3 element. On the other hand, the negative prescribed strain gradient in Case 4 results in lower stiffness values which indicate that a weakening mechanism is captured and it gets even lower for Case 6 as the value of the prescribed strain increases. 
Table 2: Bar Stiffness comparison for Copper materials of Table 1 and average Young's modulus value of $111 \mathrm{GPa}: k^{c}$ (Classical Elastic Case) with $k^{g 1} \& k^{g 2}$ (Gradient Elastic Cases) and imposed strain of $\varepsilon_{0}$ : GET bar element length $\left(I_{o}=10 \mathrm{~mm}\right), A=4 \mathrm{~mm} 2$

\begin{tabular}{|c|c|c|c|c|c|c|c|}
\hline \multicolumn{8}{|c|}{ Stiffness Value Comparison for Classical \& Gradient Elastic Element Cases $1 \& 2,\left(I_{o}=10 \mathrm{~mm}\right)$} \\
\hline $\begin{array}{l}\text { Material \& } \\
\text { Source }\end{array}$ & $\begin{array}{c}\text { Bar Stiffness } \\
\text { Values } \boldsymbol{k}^{c}\end{array}$ & $\begin{array}{c}\text { Bar Stiffness } \\
\text { Values } k^{g 1} \\
\varepsilon_{0}=0.0\end{array}$ & $\begin{array}{c}\text { Bar Stiffness } \\
\text { Values } \boldsymbol{k}^{g 2} \\
\varepsilon_{0}=0.005\end{array}$ & $\begin{array}{c}\text { Bar Stiffness } \\
\text { Values } k^{g 2} \\
\varepsilon_{0}=0.01\end{array}$ & $\begin{array}{c}\text { Bar } \\
\text { Stiffness } \\
\text { Values } \boldsymbol{k}^{g 2} \\
\varepsilon_{0}=0.05 \\
\end{array}$ & $\begin{array}{c}\text { Bar Stiffness } \\
\text { Values } k^{g 2} \\
\quad \varepsilon_{0}=1.1\end{array}$ & $\begin{array}{c}\text { Bar Stiffness } \\
\text { Values } k^{g 2} \\
\varepsilon_{0}=2.0\end{array}$ \\
\hline $\mathrm{M} 1(\ell=4.0)$ & 44400 & 44417.8 & 44416.9 & 44416.0 & 44408.9 & 44222.3 & 44062.4 \\
\hline $\mathrm{M} 6(\ell=5.84)$ & 44400 & 44425.9 & 44424.6 & 44423.4 & 44413.0 & 44140.5 & 43907.0 \\
\hline M3 $(\ell=6.0)$ & 44400 & 44426.7 & 44425.3 & 44424.0 & 44413.3 & 44133.4 & 43893.5 \\
\hline M5 $(\ell=12.0)$ & 44400 & 44453.3 & 44450.7 & 44448.0 & 44426.7 & 43866.6 & 43386.5 \\
\hline$M 4(l=20.0)$ & 44400 & 44489.0 & 44484.5 & 44480.1 & 44444.5 & 43510.2 & 42709.4 \\
\hline
\end{tabular}

Table 3: Bar Stiffness comparison for Copper materials of Table 1 and average Young's modulus value of $111 \mathrm{GPa}: \mathrm{k}^{\mathrm{c}}$ (Classical Case) with $\mathrm{k}^{\mathrm{g} 1}$ to $\mathrm{k}^{\mathrm{g} 6}$ (GET Cases) with imposed strain $\varepsilon_{0}$, GET bar element length $I_{o}=10 \mathrm{~mm}, A=4 \mathrm{~mm}^{2}$

\begin{tabular}{|c|c|c|c|c|c|c|c|}
\hline \multicolumn{8}{|c|}{ Stiffness Value Comparison for Classical \& GET Bar Element Cases 1 to $6\left(I_{o}=10 \mathrm{~mm}\right)$} \\
\hline Material \& Source & $\begin{array}{c}\text { Bar } \\
\text { Stiffness } \\
\text { Values } k^{c}\end{array}$ & $\begin{array}{c}\text { Bar Stiffness } \\
\text { Values k } \\
\varepsilon_{0}=0.0\end{array}$ & $\begin{array}{c}\text { Bar Stiffness } \\
\text { Values kg2 } \\
\varepsilon_{0}=0.005\end{array}$ & $\begin{array}{c}\text { Bar Stiffness } \\
\text { Values } k^{\mathrm{g} 3} \\
\varepsilon_{0}=0.0\end{array}$ & $\begin{array}{c}\text { Bar } \\
\text { Stiffness } \\
\text { Values } \mathbf{k}^{\mathrm{g} 4} \\
\varepsilon_{0}=\mathbf{0 . 0}\end{array}$ & $\begin{array}{c}\text { Bar } \\
\text { Stiffness } \\
\text { Values } k^{g 5} \\
\varepsilon_{0}=0.005\end{array}$ & $\begin{array}{c}\text { Bar } \\
\text { Stiffness } \\
\text { Values } \mathbf{k}^{\mathrm{g} 6} \\
\varepsilon_{0}=\mathbf{0 . 0 0 5}\end{array}$ \\
\hline $\mathrm{M} 1: \ell=4 \mu \mathrm{m}$ & 44400 & 44417.8 & 44416.9 & 44595.4 & 44240.1 & 44594.5 & 44239.2 \\
\hline M6: $\ell=5.84 \mu \mathrm{m}$ & 44400 & 44425.9 & 44424.6 & 44685.4 & 44166.48 & 44684.1 & 44165.2 \\
\hline M3: $\ell=6 \mu \mathrm{m}$ & 44400 & 44426.7 & 44425.3 & 44691.9 & 44160.1 & 44679.9 & 44158.8 \\
\hline M5: $\ell=12 \mu \mathrm{m}$ & 44400 & 44453.3 & 44450.7 & 44986.8 & 43919.90 & 44984.1 & 43917.2 \\
\hline $\mathrm{M} 4: \ell=20 \mu \mathrm{m}$ & 44400 & 44489.0 & 44484.5 & 45378.8 & 43599.2 & 45374.3 & 43594.7 \\
\hline
\end{tabular}

Another important observation from Table 2 is that for prescribed strains $\varepsilon_{0}<0.05$ the bar stiffness of Case 2 decreases as $l$ decreases from $l=20.0 \mu \mathrm{m}$ to $4.0 \mu \mathrm{m}$. But at higher strain values $\varepsilon_{0}>1.1$ the reverse is the case, the stiffness value increases as $\ell$ decreases from $\ell=20.0 \mu \mathrm{m}$ to $4.0 \mu \mathrm{m}$ since further heterogeneity is induced in the material. This reverse behavior at $\varepsilon_{0}>1.1$ agrees with the strengthening effect of strain gradient observed to be more pronounced for smaller sized microstructure at high strain due to the induced heterogeneity and an increase in the density of the GND. Dai and Bai (1998) and Dai et al. (2001) observed that GND density is controlled by the particle diameter and the smaller the size the higher the density and thus the strengthening due to strain gradient. Geers et al. (1999) also observed that by increasing local strains, the nonlocal interaction and the gradient terms become more important. A similar behavior occurs with Cases 4 and 6 for negative imposed strain gradient, the stiffness increases as $l$ decreases from $\ell=20.0 \mu \mathrm{m}$ to $4.0 \mu \mathrm{m}$ even at zero prescribed strain value $\varepsilon_{0}=0$. This is because though the negative strain gradient results in a weakening effect, the heterogeneity induced in the material during deformation by this prescribed negative strain gradient produces this strengthening behavior in materials with smaller values of $\ell$.

Thus, as expected the following three GTM bar element types (BE) can be identified from coupling Cases 1 \& 2 GTM (BE1), Cases 3 \& 5 - GTM (BE2) and Cases 4 \& 6 - GTM (BE3):

1. GTM BE1: Coupling of Cases $1 \& 2$ captures the typical microstructure deformation at the node of the bar element based on the average condition around the neighbourhood in addition to the effect of a prescribed strain. From the conditions of Section 2.4, this describes a homogeneous matrix with the gradient effect captured.

2. GTM BE2: Coupling of Cases $3 \& 5$ captures the microstructure deformation at the node due to the additional strengthening effect of a positive prescribed strain gradient resulting in the deformation being smaller than the neighbourhood thus a stiffer bar and strengthening effect that depends on the value of the prescribed strain. From the conditions of Section 2.4, this describes a strong domain in a heterogeneous material.

3. GTM BE3: Coupling of Cases $4 \& 6$ captures the microstructure deformation at the node due to the additional weakening effect of a negative prescribed strain gradient with the deformation higher at that point than the average of its neighbours, resulting in a reverse effect different from that of GTM BE2, hence a less stiff bar and weakening effect which also depends on the initial prescribed strain. Based on the conditions of Section 2.4, this describes a weak domain in a heterogeneous material. 


\subsection{Modelling of Strengthening and Weakening Microstructure Deformation Mechanism with GTM Bar Elements}

Materials combined with different stiffnesses in a heterogenous material can achieve stiffness greater than (or less than) that of any of the constituents Wu and Zhu (2017). In the strengthening of materials with reinforcement like in fibre-reinforced composite materials which consist of fibres with high strength and modulus embedded in a matrix both the fibres and matrix retain their physical and chemical identities and the new material has a combination of properties that cannot be achieved with either of the constituents acting alone.

The microstructure behavior of composite materials with such strengthening mechanism is examined here using the GTM bar elements. Since no strong weakening effect is indicated, only GTM BE1 and GTM BE2 are considered using the short glass fibre-reinforced polypropylene SGFPP of Geers et al. (1999) and unreinforced polypropylene matrix (UPM) material. The elastic material characteristics of SGFPP under classical tensile tests led to the strain equal to $1.1 \%$ and the Young's modulus E equal to $3200 \mathrm{MPa}$ which is close to the modulus of the unreinforced polypropylene matrix (UPM) material (1500-2000MPa). The characteristic internal length was obtained with a range of $c=\ell^{2}$ values 1 to 5 mm². The gradient elastic bar element length $l_{0} 100 \mathrm{~mm}$ and cross-sectional area $A=7 \mathrm{~mm}^{2}$ are assumed and from Eq. 6, Table 4 gives the stiffness value of the SGFPP with the classical elastic case obtained as $k^{c}=224.0$ while the GTM cases $k^{B E 1}$ and $k^{B E 2}$ give extremely different stiffness values as the ratio $\gamma=I_{0} / \ell$ changes from $20<\gamma<50$.

Table 4: Bar Stiffness comparison of Short glass fibre-reinforced polypropylene SGFPP with the characteristic internal length values $\mathrm{c}$ (where $\ell=\mathrm{Vc}$ ) between $1 \mathrm{~mm}^{2}$ and $5 \mathrm{~mm}^{2}$ and average Young's modulus value of $3200 \mathrm{MPa}$, Gradient elastic bar element length $I_{0}$ $100 \mathrm{~mm}$ and $\mathrm{A}=7 \mathrm{~mm}^{2}, \beta=1.2: k^{c}=224.0$ (Classical Case) with $k^{B E 1}$ and $k^{B E 2}$ (GTM Cases).

\begin{tabular}{|c|c|c|c|c|c|c|c|c|c|c|}
\hline \multicolumn{11}{|c|}{ Stiffness Value Comparison for Classical Case $k^{c}=224.0 \&$ GTM Bar Element Types $1 \& 2$ for SGFPP $I_{o}=100 \mathrm{~mm}$} \\
\hline \multirow{2}{*}{$\begin{array}{l}\text { SGFPP } \\
c\left(\ell^{2}\right)\end{array}$} & \multicolumn{4}{|c|}{ GTM BET1 $\left(k^{B E 1}\right)$} & \multicolumn{6}{|c|}{ GTM BET2 ( $\left.\left.k^{B E 2}\right)\right)$} \\
\hline & $\begin{array}{c}k^{\mathrm{BE} 1} \\
\left(\varepsilon_{\mathrm{o}}=0.0\right)\end{array}$ & $\begin{array}{c}k^{B E 1} \\
\left(\varepsilon_{o}=0.011\right)\end{array}$ & $\begin{array}{c}k^{\mathrm{BE} 1} \\
\left(\varepsilon_{\mathrm{o}}=0.5\right)\end{array}$ & $\begin{array}{c}k^{\mathrm{BE} 1} \\
\left(\varepsilon_{\mathrm{o}}=1.1\right)\end{array}$ & $\begin{array}{c}k^{B E 2} \\
\left(\varepsilon_{0}=0.0\right)\end{array}$ & $\begin{array}{c}k^{B E 2} \\
\left(\varepsilon_{0}=0.011\right)\end{array}$ & $\begin{array}{c}k^{\text {BE2 }} \\
\left(\varepsilon_{0}=0.5\right)\end{array}$ & $\begin{array}{c}k^{\mathrm{BE} 2} \\
\left(\varepsilon_{\mathrm{o}}=1.1\right)\end{array}$ & $\begin{array}{c}k^{\mathrm{BE} 2} \\
\left(\varepsilon_{\mathrm{o}}=1.5\right)\end{array}$ & $\begin{array}{c}k^{\mathrm{BE} 2} \\
\left(\varepsilon_{\mathrm{o}}=2.1\right)\end{array}$ \\
\hline $1 \mathrm{~mm}^{2}$ & 226.263 & 223.774 & 113.131 & -22.6263 & 414.815 & 412.326 & 301.684 & 165.926 & 75.4209 & -60.3367 \\
\hline $2 \mathrm{~mm}^{2}$ & 227.213 & 223.679 & 66.5492 & -126.248 & 494.987 & 491.452 & 334.323 & 141.526 & 12.9945 & -179.802 \\
\hline $4 \mathrm{~mm}^{2}$ & 228.571 & 223.543 & 0.0 & -274.286 & 609.524 & 604.495 & 380.952 & 106.667 & -76.1905 & -350.476 \\
\hline $5 \mathrm{~mm}^{2}$ & 229.123 & 223.488 & -27.0443 & -334.446 & 656.07 & 650.434 & 399.902 & 92.5006 & -112.434 & -419.835 \\
\hline
\end{tabular}

By modelling SGFPP with GTM BE1 with $\varepsilon_{0}=0$ and $c=1 \mathrm{~mm}^{2}$ the average condition in the neighborhood of the bar is captured and the stiffness $k^{B E 1}=226.263$. This implies that SGFPP is modelled as a gradient enhanced homogeneous matrix. Whereas using GTM BE2 implies that a stronger domain different from the SGFPP is modelled with a predicted stiffness value $k^{B E 2}=414.815$. This is higher than that of $k^{c}$ and $k^{g T 1}$ since the strengthening strain gradient is prescribed. For each $\mathrm{c}$ value from 1 to $5 \mathrm{~mm}^{2}$ the stiffness values of GTM BE2 closest to GTM BET1 occur at $\varepsilon_{0}=1.0$ (not shown in the table) with the following corresponding values 226.263, 227.213, 227.948, 228.571 and 229.123 respectively. Thus the weakening effect of the imposed strain on the material and the effect of the stronger domain of the heterogeneous material are captured and shown to depend on the dimension of $\mathrm{c}\left(\ell^{2}\right)$. In two-phase metal matrix composites (MMCp) experimental investigations demonstrated that the mechanical behavior is dependent on the reinforcement particle size Ling et al. (1994) and Lloyd (1994).

To verify the implication of the predicted strengthening mechanism captured with GTM BE2, the homogeneous matrix (UPM) used to obtain SGFPP is considered. In Table 5 the stiffness values is obtained for both GTM BE1 and GTM BE2 representation of UPM material based on its Young's modulus of $1750 \mathrm{MPa}$. As expected, the stiffness value of the gradient enhanced cases $k^{B E 1}$ and $k^{B E 2}$ vary as the characteristic internal length changes from $1 \mathrm{~mm}^{2}$ to $5 \mathrm{~mm}^{2}$ while the classical elastic case $k^{c}$ is fixed at 122.514 . With GTM BE1 for $\varepsilon_{0}=0$ and $\mathrm{c}=1 \mathrm{~mm}^{2}$ the stiffness value $k^{B E 1}$ of the gradient enhanced homogeneous model of UPM is 123.737 and it captures the average condition in the matrix while GTM BE2 captures a strenghening effect of a stronger domain and predicts the stiffness value $k^{B E 2}$ to be 226.852 . 
Table 5: Bar Stiffness of unreinforced polypropylene matrix UPM average (Young's modulus value of $1750 \mathrm{MPa}$ ) based on the characteristic internal length values $\mathrm{c}$ (where $\ell=\sqrt{c}$ ) between $1 \mathrm{~mm}^{2}$ and $5 \mathrm{~mm}^{2}$ and, GTM bar element length $100 \mathrm{~mm}$ and $\mathrm{A}=7 \mathrm{~mm}^{2}, B=1.2: k^{c}=122.514$ (Classical Case) with $k^{B E 1}$ and $k^{B E 2}$ (GTM Cases).

\begin{tabular}{|c|c|c|c|c|c|c|c|c|c|c|}
\hline \multirow[b]{3}{*}{$\begin{array}{l}\text { SGFPP } \\
\mathrm{c}\left(\ell^{2}\right)\end{array}$} & \multicolumn{10}{|c|}{ Stiffness Value for Unreinforced Polypropylene Matrix (UPM) using GET Bar Element Types $1 \& 2$ for UPM lo = 100mm } \\
\hline & \multicolumn{4}{|c|}{ GTM BET1 $\left(k^{B E 1}\right)$} & \multicolumn{6}{|c|}{ GTM BET2 ( $\left.k^{B E 2}\right)$} \\
\hline & $\begin{array}{c}k^{\mathrm{BE} 1} \\
\left(\varepsilon_{0}=0.0\right)\end{array}$ & $\begin{array}{c}k^{\mathrm{BE} 1} \\
\left(\varepsilon_{0}=0.011\right)\end{array}$ & $\begin{array}{c}k^{\mathrm{BE} 1} \\
\left(\varepsilon_{\mathrm{o}}=0.5\right)\end{array}$ & $\begin{array}{c}k^{\mathrm{BE} 1} \\
\left(\varepsilon_{0}=1.1\right)\end{array}$ & $\begin{array}{c}k^{\mathrm{BE2}} \\
\left(\varepsilon_{0}=0.0\right)\end{array}$ & $\begin{array}{c}k^{B E 2} \\
\left(\varepsilon_{0}=0.011\right)\end{array}$ & $\begin{array}{c}k^{\mathrm{BE2}} \\
\left(\varepsilon_{0}=0.5\right)\end{array}$ & $\begin{array}{c}k^{\mathrm{BE} 2} \\
\left(\varepsilon_{0}=1.1\right)\end{array}$ & $\begin{array}{c}k^{\mathrm{BE2}} \\
\left(\varepsilon_{0}=1.5\right)\end{array}$ & $\begin{array}{c}k^{\mathrm{BE2} 2} \\
\left(\varepsilon_{0}=2.1\right)\end{array}$ \\
\hline $1 \mathrm{~mm}^{2}$ & 123.737 & 122.376 & 61.8687 & -12.3737 & 226.852 & 225.491 & 164.983 & 90.7407 & 41.2458 & $\begin{array}{c}- \\
32.9966\end{array}$ \\
\hline $2 m m^{2}$ & 124.257 & 122.324 & 36.3941 & -69.0417 & 270.696 & 268.763 & 182.833 & 77.3969 & 7.10639 & $\begin{array}{c}- \\
98.3294\end{array}$ \\
\hline $3 \mathrm{~mm}^{2}$ & 124.659 & 122.284 & 16.7012 & -112.848 & 304.589 & 302.214 & 196.631 & 67.0816 & $\begin{array}{c}- \\
19.2848\end{array}$ & $\begin{array}{c}- \\
148.834\end{array}$ \\
\hline $4 \mathrm{~mm}^{2}$ & 125.0 & 122.25 & 0.0 & -150.0 & 333.333 & 330.583 & 208.333 & 58.3333 & $\begin{array}{c}- \\
41.6667\end{array}$ & $\begin{array}{c}- \\
191.667\end{array}$ \\
\hline $5 \mathrm{~mm}^{2}$ & 125.302 & 122.22 & -14.7899 & -182.9 & 358.788 & 355.706 & 218.696 & 50.5863 & $\begin{array}{c}- \\
61.4871\end{array}$ & $\begin{array}{c}- \\
229.597\end{array}$ \\
\hline
\end{tabular}

A comparison of the stiffness $k^{B E 1}=\mathbf{2 2 6 . 2 6 3}$ obtained for SGFPP as a gradient enhanced homogeneous matrix using GTM BET1 in Table 4 and $k^{B E 2}=226.852$ obtained for UPM using GTM BE 2 in Table 5 shows that the latter actually predicts the stiffness of SGFPP. Though GTM BE 2 represents a stronger domain in the homogeneous matrix of UPM, it actually also predicts the strengthening of UPM as an heterogeneous material as is the case with SGFPP. Moreover, this stiffness value is even comparable to the classical case $k^{c}=224.0$ obtained for SGFPP. This indicates that the stiffness of UPM as a composite material strengthened with the short glass fibres SGF reinforcement to obtain SGFPP was qualitatively predicted with GTM BET2 based on using the Young's modulus of UPM and prescribing the strengthening effect as positive strain gradient. The effectiveness of this result is further elaborated in Figure 11 for the different characteristic internal length values. Thus as shown in Table 4, using GTM BE2 and the Young's modulus of SGFPP predicts a different domain and material response which is a stronger one than SGFPP. This is a qualitative verification of the capacity of GTM BE2 to effectively predict and model strengthening mechanisms in composite materials. 


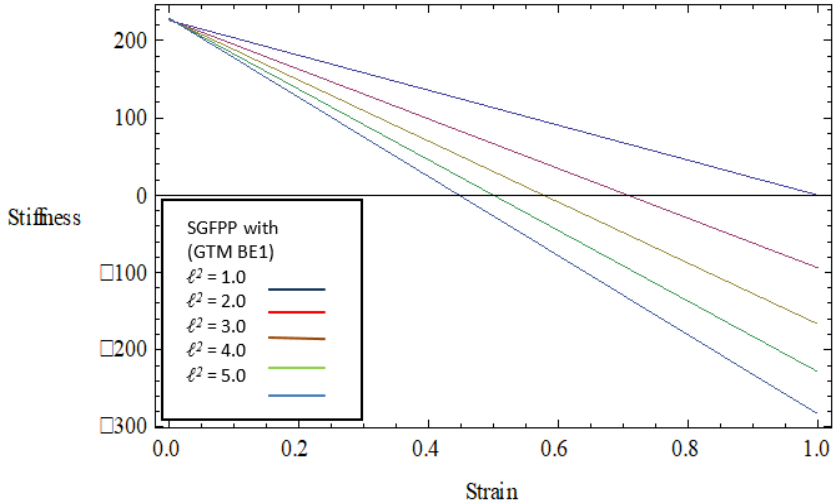

(a)

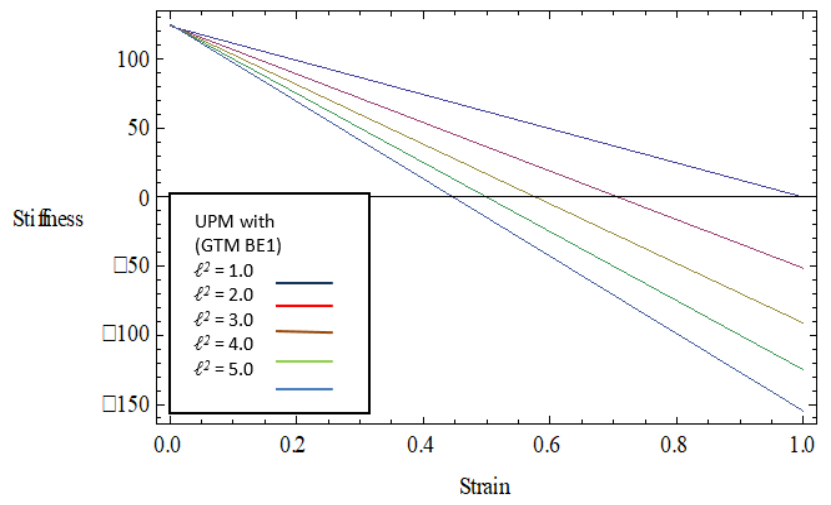

(c)

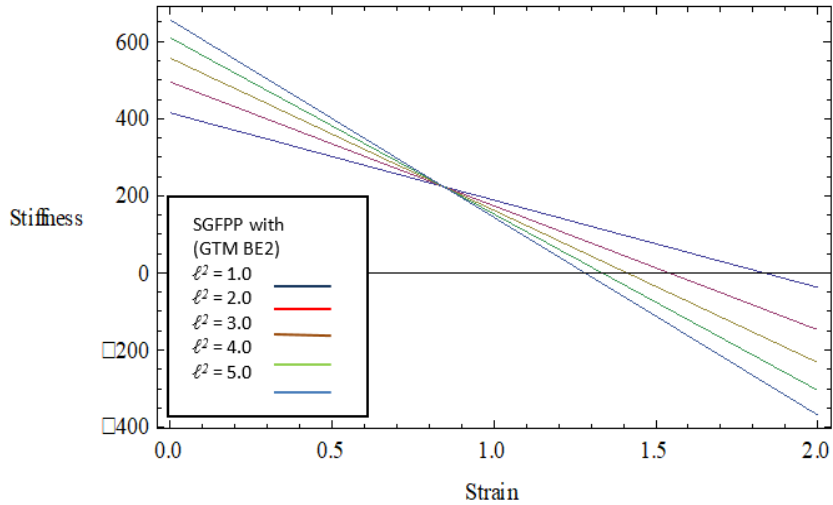

(b)

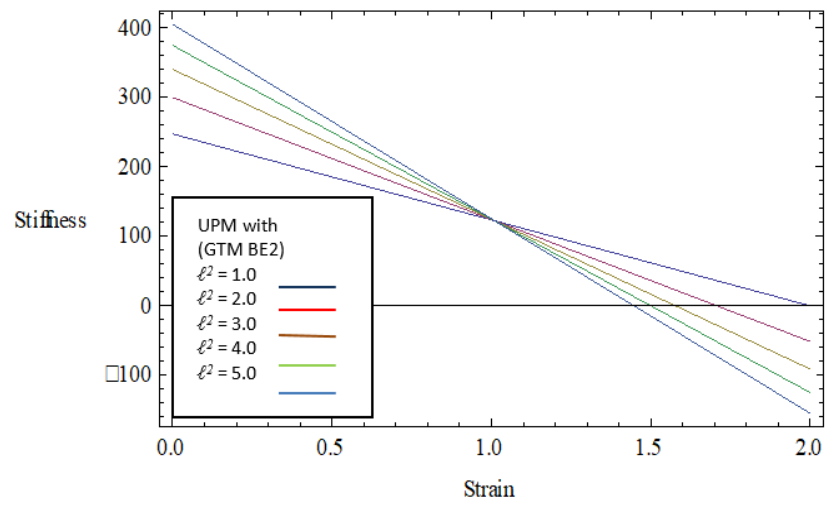

(d)

Figure 11: Bar Stiffness comparison: a. SGFPP with GTM BE1; b. SGFPP with GTM BE2; c. UPM with GTM BE1; d. UPM with GTM BE2.

For instance, it is clearly shown that GTM BE2 model of UPM captures the underlying strengthening phenomena not captured with GTM BE1 model of SGFPP which only considers the average condition around the neighbourhood and models SGFPP as a homogeneous matrix. The effect of the strengthening captured is evident from the higher stiffness values obtained for the different characteristic internal lengths in Table $5\left(\mathrm{c}=2, k^{B E 2}=270.696\right),\left(\mathrm{c}=3, k^{B E 2}=304.589,(\mathrm{c}=4\right.$, $\left.k^{B E 2}=333.333\right),\left(\mathrm{c}=5, k^{B E 2}=358.788\right)$ as compared to those obtained with GTM BE 1 model of SGFPP in Table 4 (c=2, $\left.k^{B E 1}=227.213\right),\left(\mathrm{c}=3, k^{B E 1}=227.948, \quad\left(\mathrm{c}=4, k^{B E 1}=228.571\right),\left(\mathrm{c}=5, k^{B E 1}=229.123\right)\right.$. Moreover, though the imposed strain decreases the stiffness value in Tables 4 and 5, nevertheless for GTM BE1 model of SGFPP at prescribed strain $\varepsilon_{0}=0.011$ and $c=1 \mathrm{~mm}^{2}$, the stiffness value is obtained as 223.774 . The first zero stiffness value observed occurs at $\varepsilon_{0}=0.5$ for $c=4 \mathrm{~mm}^{2}$ while at $\varepsilon_{0}=1.1$ the stiffness values are already negative for all $c$ values. However, the GTM BE2 model of UPM which predicts the values for SGFPP gives a stiffness value of 225.491 for the same $c$ and $\varepsilon_{\mathrm{o}}$ values, with no zero stiffness values observed at $\varepsilon_{0}=0.5$. The negative stiffness values observed occured much later at prescribed strain $\varepsilon_{0}=1.5$ for $\mathrm{c}=3 \mathrm{~mm}^{2}, 4 \mathrm{~mm}^{2}$ and $5 \mathrm{~mm}^{2}$. This is buttressed in Figure 11. These higher stiffness values observed for GTM BE2 model of UPM (which is the prediction of SGFPP) regardless of the prescribed strain is a result of the effect of a stronger domain been captured rather than only the average condition. Consequently, it confirms that GTM BE1 can be used to model a homogeneous matrix while GTM BE2 can be used as a model to predict the response of a matrix strengthened with stronger domains (heterogeneous).

Furthermore, although the positive imposed strain gradient and the imposed strain represent different mechanisms, at increasing imposed strain a change in behaviour occurs at different transition values for models GTM BE1 and 2 as the size of the characteristic internal length varies from $1 \mathrm{~mm}^{2}$ to $5 \mathrm{~mm}^{2}$. For GTM BE1 model of SGFPP at lower imposed strain of $\varepsilon_{0}<0.011$ (which is actually the strain observed for SGFPP during the tensile test of Geers et al. (1999)) the bars are stiffer for larger characteristic internal length but a reverse is the case beyond $\varepsilon_{0}>0.011$ with smaller becoming stiffer. This is also the case using GTM BE1 model of UPM. This transition can be linked to that observed after the yield point is reached and plastic deformation and strain hardening takes place. On the other hand, for GTM BE2 models of both SGFPP and UPM this change is observed at higher imposed strains $\varepsilon_{0}>1.1$. This is because as strain increases heterogeneity is increased. The effect of the increase in strain is shown more obviously in Figure 11. 
In the classical truss modelling of the influence of strain gradient on Reinforced Concrete R.C columns Akintayo et al. (2000) observed that for the monotonic loading case the strain gradient over the RC section increased the ductility of the R.C column since the failure occurred for larger peak concrete compressive strain. However, it couldn't reveal the effect of changes in the microstructure dimension and response. In a microscopic view, high strain gradient will promote the development of the localized plastic deformation and the composite with the smallest particles is of the highest flow stress and its dissipated plastic work is greater than cases of large particles Dai et al. (2004). These observations are expected since with increasing applied strain the deformation process can be classified into three stages for a heterogeneous material. In the first stage both the soft and hard domains deform elastically while in the second stage, to produce plastic strain the soft domain will start dislocation slip while the hard domain will remain elastic which creates a mechanical incompatibility and a plastic strain gradient in the soft domain near the interface. To accommodate this strain gradient, geometrically necessary dislocations are produced, which will result in the softer domain appearing stronger. At the final stage, both domains deform plastically with the soft domain sustaining much higher strain than the hard domains, producing the so-called strain partitioning Park et al. (2014), Tasan et al. (2014) Han et al. (2014), Wang et al. (2015) and Yang et al. (2016a). When neighboring domains sustain different plastic strains, strain gradients are expected to exist near the domain boundaries in both the soft and hard domains. These strain gradients become larger with increasing strain partitioning, and consequently produce back-stress work hardening Wu and Zhu (2017). In metals, the back-stress work hardening assists with the prevention of necking and thus improving ductility, which is the primary reason why dual-phase steel has extraordinary work hardening, and consequently high ductility Park et al. (2014), Han et al. (2014), and Yang et al. (2016b). On the other hand for RC the cyclic loading case showed that after the first half loading, because of the permanent plastic strains, the strain gradients could not prevent the early cracking of diagonal concrete bars and these, in turn did not prevent the premature buckling of internal vertical concrete struts and the subsequent global instability of the RC column Akintayo et al. (2000).

Another important observation is the effect of the particle size of UPM which could range between 0.03 to $1.0 \mu \mathrm{m}$, Paik and Kar (2009), Lotti et al (2000): from Section 3.1 the $\ell$ value range for the unreinforced homogeneous matrix should fall within the micrometer range. However, this was not considered in Table 5 for GTM BE1 since the focus was GTM BE2. But in Table 6 based on an assumed $\ell$ range of $0.9 \mu \mathrm{m}-3.0 \mu \mathrm{m}$ and using GTM BE1 the stiffness values are obtained as the strain changes from $\varepsilon_{0}=0$ to $\varepsilon_{0}=1.1$. It should be noted that similar to the results in Section 4.1 for copper materials because of the large ratio between the bar length and the characteristic internal length $\gamma=I_{0} / \ell=120,000$, the values obtained are comparable to those of the classical case since the magnitude of strain gradients is negligible for bulk materials but is pronounced at such small-scale. On the other hand for the SGFPP composite material the composition of the unit volume changes due to the fibers introduced into the UPM matix which is reflected in the higher values of $\ell$ obtained in Geers et al. (1999) and used in Table 4 and 5 . As shown in this section for any significant differences to be captured by GTM the value of $I_{0}$ should be comparable to $\ell$ : in this case $\gamma<100$.

Table 6: Bar Stiffness of unreinforced polypropylene matrix average (Young's modulus value of $1750 \mathrm{MPa}$ ), $\mathrm{c}$ (where $\ell=\mathrm{V} c$ ) between $0.81 \mu \mathrm{m}^{2}$ and $9 \mu \mathrm{m}^{2}$ and, GTM bar element length $100 \mathrm{~mm}$ and $\mathrm{A}=7 \mathrm{~mm}^{2}: k^{c}=122.514$ (Classical Case) with $k^{B E 1}$ (GTM Cases).

\begin{tabular}{|c|c|c|c|c|}
\hline \multicolumn{5}{|c|}{ Stiffness Value for Unreinforced Polypropylene Matrix (UPM) using GTM BET1 $k^{B E 1}$ for UPM lo $=100 \mathrm{~mm}$} \\
\hline SGFPP & $k^{\mathrm{BE} 1}$ & $k^{\mathrm{BE} 1}$ & $\mathbf{k}^{\mathrm{BE} 1}$ & $\mathbf{k}^{\mathrm{BE} 1}$ \\
\hline $\mathrm{c}\left(\ell^{2}\right)$ & $\left(\varepsilon_{0}=0.0\right)$ & $\left(\varepsilon_{0}=0.011\right)$ & $\left(\varepsilon_{0}=0.5\right)$ & $\left(\varepsilon_{0}=1.1\right)$ \\
\hline $0.81 \mu \mathrm{m}^{2}$ & 122.611 & 122.501 & 122.446 & 122.446 \\
\hline $1.0 \mu \mathrm{m}^{2}$ & 122.624 & 122.501 & 122.44 & 122.44 \\
\hline $4.0 \mu \mathrm{m}^{2}$ & 122.747 & 122.502 & 122.38 & 122.38 \\
\hline $9.0 \mu \mathrm{m} 2$ & 122.871 & 122.503 & 122.32 & 122.32 \\
\hline
\end{tabular}

For weakening mechanisms, it is well known that the introduction of pores into a structural material will dramatically change its mechanical properties of stiffness, strength, and fatigue resistance. In addressing the modelling of weakening mechanisms with the GTM bar elements, the study of Zou et al. 2016 is considered based on a porous metal fiber/powder sintered composite sheet (PMFPSCS) developed by sintering a mixture of a porous metal fiber sintered sheet (PMFSS) and copper powders with particles of spherical shape. The dimensions of the PMFPSCS specimen were $120 \mathrm{~mm}$ in length, $15 \mathrm{~mm}$ in width and $2 \mathrm{~mm}$ in thickness. The copper particles of the matrix were found to be between the range of $25-100 \mu \mathrm{m}$ and the copper fibres were $15 \mathrm{~mm}$ in length. When the porosity of the PMFSS was constant, the amount of copper powder increased with copper particle size decreasing, thus the strength of the PMFPSCS increased. As earlier indicated for the purpose of this study, the characteristic internal length values considered for the 
PMFPSCS composite is assumed to fall between $1 \mathrm{~mm}^{2} \leq \ell^{2} \geq 9 \mathrm{~mm}^{2}$. The dimensions of the bar element is taken as in the experiment. As expected the stiffness value for the classical case $k^{c}$ is fixed at 925.0. In Table 7 it can also be seen that at $\varepsilon_{\mathrm{o}}=0$ the GTM BE1 stiffness value $k^{g T 1}$ of the material with $\mathrm{c}=1 \mathrm{~mm}^{2}$ is 932.773 and the GTM BE2 stiffness value $k^{B E 2}$ is 1865.55 which implies a stronger domain than the PMFPSCS is captured.

Table 7: Bar Stiffness comparison of porous metal fiber powder sintered composite sheet (PMFPSCS) with the characteristic internal length values $\ell^{2}=c=1.0,2.0,3.0,4.0,5.0, \mathrm{~mm}^{2}$, Young's modulus value of $3700 \mathrm{MPa}$, imposed strain of $\varepsilon_{0}$, GTM bar element length $I_{o}=120 \mathrm{~mm}$ and cross-sectional area $A=30 \mathrm{~mm}^{2}, k^{c}=925.0$ (Classical Case) with $k^{B E 1}, k^{g B E 2}$ and $k^{B E 3}$ (GET Cases).

\begin{tabular}{|c|c|c|c|c|c|c|c|c|c|c|c|c|}
\hline \multirow[b]{3}{*}{$\begin{array}{c}\text { PMFPSCS } \\
\text { c }\left(\ell^{2}\right)\end{array}$} & \multicolumn{12}{|c|}{ Stiffness Value Comparison for PMFPSCS with Classical \& GTM Bar Element Types 1, 2 \& 3 for PMFPSCS $\left(I_{o}=120 \mathrm{~mm}\right)$} \\
\hline & \multicolumn{4}{|c|}{ GTM BE1 $\left(k^{B E 1}\right)$} & \multicolumn{4}{|c|}{ GTM BE2 $\left(k^{B E 2}\right)$} & \multicolumn{4}{|c|}{ GTM BE3 $\left(k^{B E 3}\right)$} \\
\hline & $\begin{array}{c}k^{\text {BE1 }} \\
\left(\varepsilon_{0}=0.0\right)\end{array}$ & $\begin{array}{c}k^{\text {BE1 }} \\
\left(\varepsilon_{0}=0.005\right)\end{array}$ & $\begin{array}{c}k^{B E 1} \\
\left(\varepsilon_{0}=0.5\right)\end{array}$ & $\begin{array}{c}k^{\mathrm{BE} 1} \\
\left(\varepsilon_{0}=1.1\right)\end{array}$ & $\begin{array}{c}k^{\mathrm{BE} 2} \\
\left(\varepsilon_{0}=0.0\right)\end{array}$ & $\begin{array}{c}k^{\text {BE2 }} \\
\left(\varepsilon_{0}=0.005\right)\end{array}$ & $\begin{array}{c}k^{\mathrm{BE} 2} \\
\left(\varepsilon_{\mathrm{o}}=0.5\right)\end{array}$ & $\begin{array}{c}k^{B E 2} \\
\left(\varepsilon_{0}=1.1\right)\end{array}$ & $\begin{array}{c}k^{\mathrm{BE3}} \\
\left(\varepsilon_{\mathrm{o}}=0.0\right)\end{array}$ & $\begin{array}{c}k^{\mathrm{BE3}} \\
\left(\varepsilon_{0}=0.005\right)\end{array}$ & $\begin{array}{c}k^{\mathrm{BE3}} \\
\left(\varepsilon_{0}=0.5\right)\end{array}$ & $\begin{array}{c}k^{\mathrm{BE3}} \\
\left(\varepsilon_{0}=1.1\right)\end{array}$ \\
\hline $1 \mathrm{~mm}^{2}$ & 932.773 & 928.109 & 466.387 & -93.2773 & 1865.55 & 1860.88 & 1399.16 & 839.496 & $\begin{array}{c}1.43044 \times 10^{-} \\
49\end{array}$ & -4.66387 & -466.387 & -1026.05 \\
\hline $2 \mathrm{~mm}^{2}$ & 936.031 & 929.412 & 274.157 & -520.092 & 2259.78 & 2253.16 & 1597.91 & 803.656 & -387.717 & -394.336 & -1049.59 & -1843.84 \\
\hline $4 \mathrm{~mm}^{2}$ & 940.678 & 931.271 & 0. & -1128.81 & 2822.03 & 2812.63 & 1881.36 & 752.542 & -940.678 & -950.085 & -1881.36 & -3010.17 \\
\hline $5 \mathrm{~mm}^{2}$ & 942.564 & 932.025 & -111.255 & -1375.84 & 3050.2 & 3039.66 & 1996.38 & 731.8 & -1165.07 & -1175.61 & -2218.89 & -3483.47 \\
\hline
\end{tabular}

Furthermore as previously compared for SGFPP and UPM, the implication of the strengthening mechanism predicted using GTM BE2 is buttressed in Table 8 by comparing the stiffness values obtained with GTM BE1 representation of PMFSS material (which is the matrix used to obtain PMFPSCS with Young's modulus of $1700 \mathrm{MPa}$ ). In this case using GTM BE1 for the homogeneous PMFSS and by considering the porous domain as the second component of the material and the porous metal fiber as the matrix the $\ell$ values can be obtained based on Eq. 8 . Since the porous metal fiber has the fiber length of $15 \mathrm{~mm}$ and $80 \%$ porosity based on Eq. 8 the range of $\ell$ values for PMFSS is obtained as $1.5 \mathrm{~mm} \leq \ell \leq 3 \mathrm{~mm}$ which falls close to the range of $\ell^{2}=c=1.0,2.0,3.0,4.0,5.0 \mathrm{~mm}^{2}$. Hence as illustration this range of $\ell$ value is maintained. As expected, the stiffness value of the classical elastic case $k^{c}$ is fixed at 425.0 . For $\varepsilon_{0}=0$ and $\mathrm{c}=1 \mathrm{~mm}{ }^{2}$ using GTM BE1 the stiffness value for the gradient enhanced homogeneous model of PMFSS is $k^{B E 1}=428.571$ while using GTM BE2 with the same $c$ and $\varepsilon_{0}$ values predicts the stiffness value $k^{B E 2}$ to be 783.571 and captures a strenghening effect prescribed as positive strain gradient (i.e. a gradient enhanced heterogeneous model of PMFSS).

Table 8: Bar Stiffness comparison of porous metal fiber sintered sheet(PMFSS) with the characteristic internal length values $\ell^{2}=\mathrm{c}=$ $1.0,2.0,3.0,4.0,5.0 \mathrm{~mm}^{2}$, Young's modulus value of $1700 \mathrm{MPa}$, imposed strain of $\varepsilon_{0}$, GTM bar element length $I_{0}=120 \mathrm{~mm}$ and crosssectional area $A=30 \mathrm{~mm}^{2}, k^{c}=425.0$ (Classical Case) with $k^{B E 1}, k^{B E 2}$ and $k^{B E 3}$ (GET Cases)

\begin{tabular}{|c|c|c|c|c|c|c|c|c|c|c|c|c|}
\hline \multirow[b]{3}{*}{$\begin{array}{c}\text { PMFPSCS } \\
c\left(\ell^{2}\right)\end{array}$} & \multicolumn{12}{|c|}{ Stiffness Value Comparison for PMFSS Classical \& GTM Bar Element Types 1, 2 \& $3\left(I_{o}=300 \mathrm{~mm}\right)$} \\
\hline & \multicolumn{4}{|c|}{ GTM BE1 $\left(k^{B E 1}\right)$} & \multicolumn{4}{|c|}{ GTM BE2 $\left(k^{B E 2}\right)$} & \multicolumn{4}{|c|}{ GTM BE3 ( $\left.k^{B E 3}\right)$} \\
\hline & $\begin{array}{c}\mathbf{k}^{\text {BE1 }} \\
\left(\varepsilon_{0}=0.0\right)\end{array}$ & $\begin{array}{c}k^{\text {BE1 }} \\
\left(\varepsilon_{0}=0.005\right)\end{array}$ & $\begin{array}{c}k^{B E 1} \\
\left(\varepsilon_{0}=0.5\right)\end{array}$ & $\begin{array}{c}k^{B E 1} \\
\left(\varepsilon_{0}=1.1\right)\end{array}$ & $\begin{array}{c}k^{B E 2} \\
\left(\varepsilon_{0}=0.0\right)\end{array}$ & $\begin{array}{c}k^{\text {BE2 }} \\
\left(\varepsilon_{0}=0.005\right)\end{array}$ & $\begin{array}{c}k^{B E 2} \\
\left(\varepsilon_{0}=0.5\right)\end{array}$ & $\begin{array}{c}k^{B E 2} \\
\left(\varepsilon_{0}=1.1\right)\end{array}$ & $\begin{array}{c}k^{\mathrm{BE3}} \\
\left(\varepsilon_{0}=0.0\right)\end{array}$ & $\begin{array}{c}k^{\text {BE3 }} \\
\left(\varepsilon_{0}=0.005\right)\end{array}$ & $\begin{array}{c}k^{\mathrm{BE3}} \\
\left(\varepsilon_{\mathrm{o}}=0.5\right)\end{array}$ & $\begin{array}{c}k^{\text {BE3 }} \\
\left(\varepsilon_{0}=1.1\right)\end{array}$ \\
\hline $1 \mathrm{~mm}^{2}$ & 428.571 & 426.429 & 214.286 & -42.8571 & 785.714 & 783.571 & 571.429 & 314.286 & 71.4286 & 69.2857 & -142.857 & -400 \\
\hline $2 m m^{2}$ & 430.068 & 427.027 & 125.964 & -238.961 & 936.909 & 933.868 & 632.805 & 267.879 & -76.7721 & -79.8131 & -380.876 & -745.801 \\
\hline $3 \mathrm{~mm}^{2}$ & 431.224 & 427.49 & 57.7731 & -390.368 & 1053.64 & 1049.91 & 680.192 & 232.05 & -191.194 & -194.929 & -564.645 & -1012.79 \\
\hline $5 \mathrm{~mm}^{2}$ & 433.07 & 428.228 & -51.117 & -632.141 & 1240.05 & 1235.21 & 755.861 & 174.837 & -373.908 & -378.75 & -858.095 & -1620.75 \\
\hline
\end{tabular}




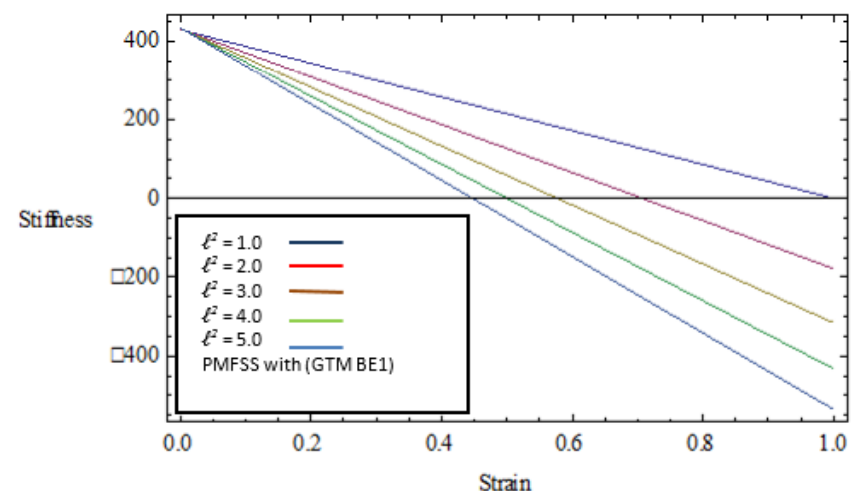

(a)

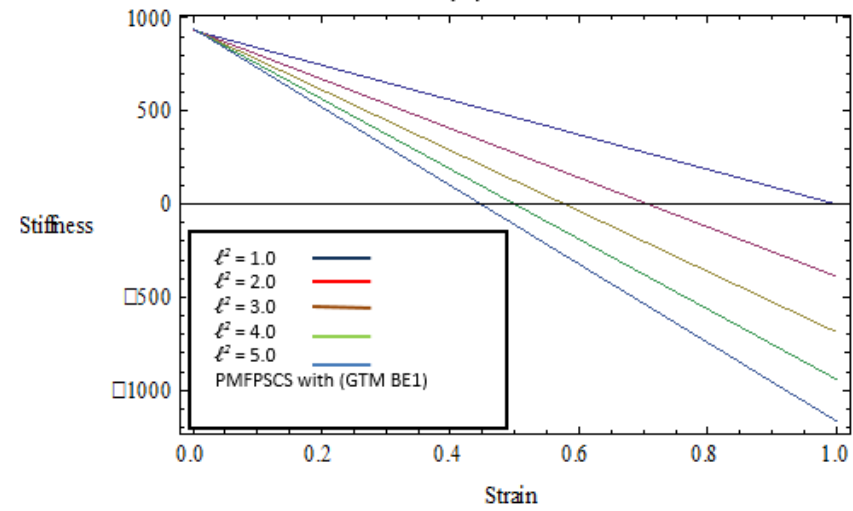

(c)

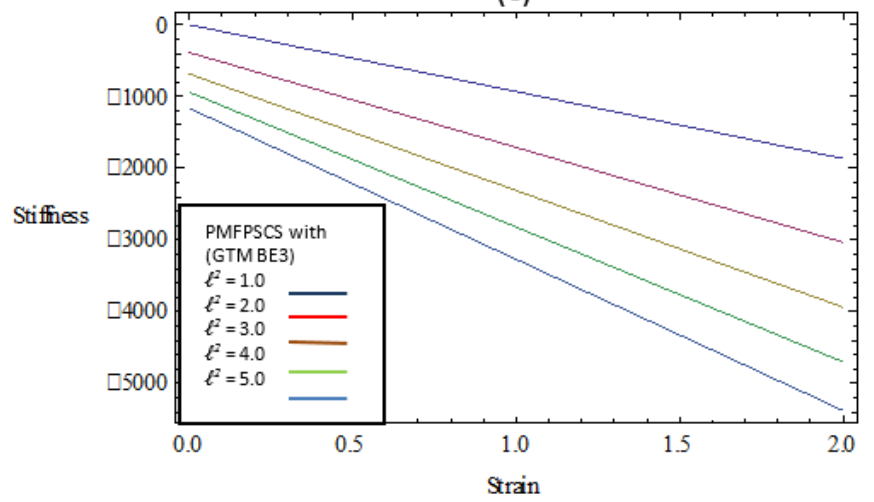

(e)

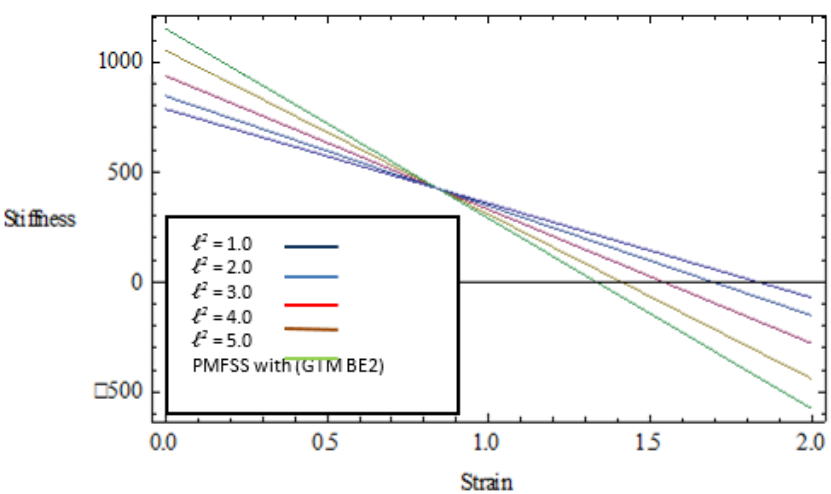

(b)

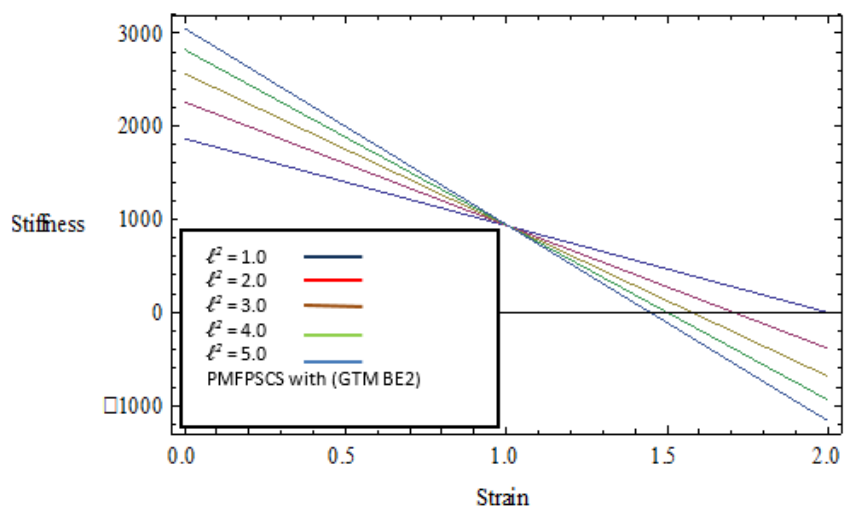

(d)

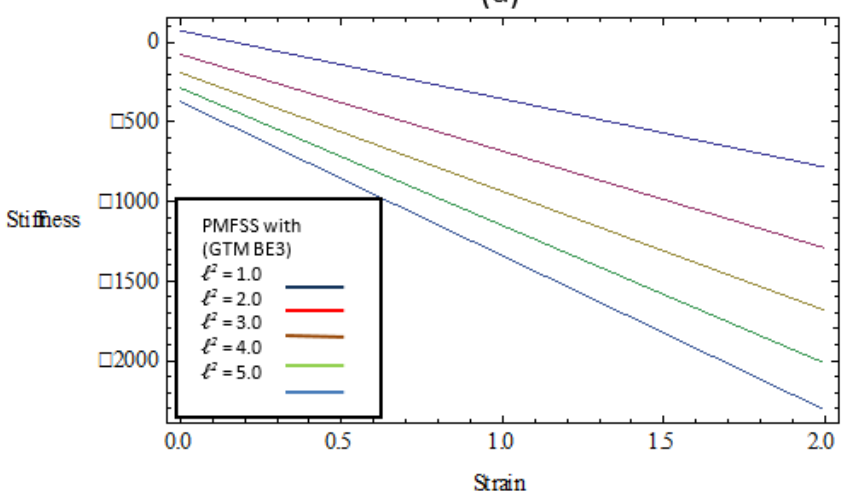

(f)

Figure 12: Bar Stiffness comparison: a. PMFSS with GTM BE1; b. PMFSS with GTM BE2; c. PMFPSCS with GTM BE1; d. PMFPSCS with GTM BE2; e. PMFPSCS with GTM BE3; f. PMFSS with GTM BE3.

However, in comparing the stiffness using GTM BE1 for PMFPSCS obtained as $k^{B E 1}=932.773$ in Table 6 , the stiffness using GTM BE2 for PMFSS with $\mathrm{c}=1 \mathrm{~mm}^{2}$ obtained as $k^{B E 2}=783.571$ and $\mathrm{c}=2 \mathrm{~mm}^{2}$ obtained as $k^{B E 2}=936.909$ in Table 8 , reveals that the latter is a better prediction of PMFPSCS. It verifies the proximity of the lower value $l=1.54 \mathrm{~mm}$ obtained in Section 3.1 and suggests $c=2 \mathrm{~mm}^{2}$ (i.e $\ell=1.414 \mathrm{~mm}$ ) as the lower limit range not $(\ell=1.54 \mathrm{~mm}$ ).

The effect of increasing strain on the behavior of the PMFPSCS at the microscale is also revealed in Table 6 and much obvious in Fig. 12. It shows that the transition value observed in Table 6 is much lower than $\varepsilon_{0}<0.5$ and falls between $0.0001<\varepsilon_{0}>0.01$ comparable to the observation of Zou et al. (2016) where the elastic stage was very short and a strain range of $0.05 \%-0.08 \%$ was observed. Moreover, Zou et al. (2016) showed the structural elastic deformation to be dominant at the initial elastic stage and at the hardening stage the plastic deformation was composed of the structural deformation and the copper fibres' plastic deformation. After the stress reached the maximum value, the PMFPSCS sample began to fracture. In Fig. $12 \mathrm{c}$ as the strain increases beyond $\varepsilon_{0}>0.0005$ this observed behavior is revealed and shown to depend on the microstructure dimension.

On the other hand for a weak domain in PMFPSCS obtained using GTM BE3 for $\mathrm{c}=1 \mathrm{~mm}^{2}$ the stiffness $k^{B E 3}$ is $1.43044 \times 10^{-49}$ in Table 7 which is very low and negative for higher values of $\mathrm{c}>2.0 \mathrm{~mm}^{2}$ (confirms $\mathrm{c}=2 \mathrm{~mm}^{2}$ to be the 
lower limit). Invariably the weakening captured does not account for any strengthening from the copper particles but the effect of a strongly weak domain. Moreover, in Table 8 based on the prediction of a weak domain in PMFSS the stiffness value obtained using GTM BE3 and $c=1 \mathrm{~mm}^{2}$ is 71.4286 and also negative for higher values of $c>2.0 \mathrm{~mm}^{2}$, which represents a weaker domain than the average PMFSS matrix. Zou et al. (2016) found that the tensile strength of the PMFPSCS depended significantly on the porosity of the PMFSS skeleton and decreased dramatically when the porosity of the PMFSS increased resulting in a weak strain-hardening effect. Thus, the extremely low and negative stiffness observed using GTM BE3 suggests the presence of a porous domain, a void, or micro-crack and that such weakening heterogeneity in materials result in negative strain gradient. Moreover, in examining the implication of these negative stiffness values at zero strain for instance, negative stiffness elements have been observed to contribute to damping behavior because they tend to assist rather than resist deformation as a result of internally stored energy (Lakes, 2001a, 2001b). In Table 7 and 8 negative stiffness is observed for GTM BE3 even at zero imposed strain for higher values of c $>2.0 \mathrm{~mm}^{2}$, which indicates it is not only due to the imposed strain but the nature of the domain modelled and the reverse effect captured. Hence, in this case the magnitude of the deformation would be large even under very small force. A domain of negative stiffness exerts a reaction force in the same direction as the deformation, which tends to help the deformation proceed further, the deforming object has positive stored energy at unstable equilibrium Lakes 2001 or is supplied with energy Thompson 1982. The effective stiffness may approach infinity, but it may be in a metastable state. This property is usually unstable, but it has been shown theoretically that inclusions of negative stiffness can be stabilized within a positive-stiffness matrix, Lakes and Drugan 2002. Though the effect of the reinforcement cannot be undermined, it is evident that the porosity of the PMFSS skeleton was the physical controlling microstructure feature of the deformation of the PMFPSCS sample. The porosity of metal fiber porous material can be as high as $98 \%$ while the pore size is smaller than $10 \mu \mathrm{m} \mathrm{Xi} \mathrm{et} \mathrm{al.} \mathrm{(2011).} \mathrm{Moreover,} \mathrm{Table} 7$ and 8 reveal that the effect of the porous or weak domain on the stiffness values is lower for smaller characteristic internal length values which is in agreement with the observation of Moore et al. (2006) for foams of small pore size.

\section{CONCLUSIONS}

In this paper, three gradient truss model bar elements (GTM BEs) which represent three typical underlying phenomena at the microstructure are presented. In a simple manner they are defined by three coupled combinations of extra non-classical boundary conditions of the derivative of displacement (strain) and higher order derivative of displacement (strain gradient) imposed at the bar support:

GTM BE1 represents the average condition around the neighbourhood of a point which could readily model the microstructure response of a homogeneous matrix with or without initial strain.

GTM BE2 represents a material domain with higher strength and stiffness at the point than the average around its neighbourhood and this will readily model the microstructure response of a stronger domain in a matrix or a reinforced heterogeneous material with or without initial strain.

GTM BE3 represents a material domain with a much weaker condition than the average around its neighbourhood and this can readily model the microstructure response of a weak domain in a heterogeneous material such as porous domains, voids, cracks or domains with negative stiffness, with or without initial strain.

The imposed strain gradient was quantified based on a simple but effective expression derived relating it to the inverse of the characteristic internal length and a characteristic deformation parameter.

Furthermore as illustration to obtain the characteristic internal length $\ell$ for use in the GTM a simple relation was derived for heterogeneous materials. The value obtained for SGFPP was predicted to be $1.9 \mathrm{~mm} \leq \ell \leq 3.83 \mathrm{~mm}$ which relatively compares to the range $1 \mathrm{~mm} \leq \ell \leq 2.236 \mathrm{~mm}$ obtained in Geers et al. (1999). For PMFPSCS it was predicted to be $1.5 \mathrm{~mm} \leq \ell \leq 3.0 \mathrm{~mm}$ not revealed in the experiment of Zou et al. (2016). Thus this suggests that a qualitative statistical-deterministic approach to obtain a range of $\ell$ values may be explored. This is examined in a forthcoming publication.

Two composite materials SGFPP and PMFPSCS examined with the bar types showed qualitative representation of the different modelled effects and revealed the influence of the heterogeneity present, increase in strain, strain gradient and characteristic internal length in strengthening and weakening mechanisms.

It should be noted that for the application of these GTM BEs issues relating to nonlinear behavior and softening consideration should be appropriately addressed.

Editor: Marco L. Bittencourt. 


\section{References}

Abu Al-Rub, R.K., Voyiadjis, G.Z. (2004). Analytical and experimental determination of the material intrinsic length scale of strain gradient plasticity theory, from micro and nano indentation experiments, International J. of Plasticity 20, 1139-1182.

Aifantis, E. C. (1984). On the microstructural origin of certain inelastic models, Transactions of ASME, J. Engng. Mat. Tech. $106,326-330$.

Aifantis, E. C. (1992). On the role of gradients in the localization of deformation and fracture. Int J Eng Sci 30:1279-1299.

Aifantis E.C. (1994). Gradient effects at macro, micro, and nano scales. J. Mech. Behav. Mat. 5: 355-375

Akintayo, F., Papadopoulos, P.G., Aifantis, E.C. (1998). Simulation of Uniaxial Compression of a Concrete Column by a Truss Model with Instability, Proc. of $5^{\text {th }}$ National Congress on Mechanics, Ioannina, Greece, Vol. 2, pp.899-906.

Akintayo, F., Papadopoulos, P.G., Aifantis, E.C. (2000). Influence of strain gradient on the ductility of a reinforced concrete column: Abnormal loading on structures: experimental and numerical modelling, Ed. K. S. Virdi 162(10). Spon Press.

Akintayo, O. T. (2011). Analytical and Numerical Study of the Behavior of Materials and Structures in Gradient Elasticity, Ph.D. Thesis, Aristotle University Thessaloniki, Greece.

Akintayo, O. T., Papadopoulos, P.G., Aifantis, E.C. (2012). A note on gradient truss models. International Review of Mechanical Engineering Vol. 6, N. 4, 5.

Akintayo, O. T. (2014a). Towards a gradient truss model Part I: Bar Element Displacement-Force Relations, International Review of Civil Engineering Vol. 5. N.1, 32-42, 1.

Akintayo, O. T. (2014b). The Effect of Microstructure Size on Bar Cross-Sectional Area Changes in Elastic Tensile Deformation, Journal on Advance Materials and Technologies, Vol. 2. N.4, 91-95, 7.

Akintayo, O. T., (2014c). Influence of Microstructure and Specimen Size ratio on the Elastic Modulus of a Material, Int. Review of Mechanical Engineering, Vol.8. N. 4, 689-701, 7.

Akintayo, O. T. (2014d). Size and Scale Dependence of Truss Models; A Gradient elasticity Approach: $5^{\text {th }}$ Int. Conference on Computational Methods 28-30 th July, Cambridge, England.

Akintayo, O. T. (2018). Towards a gradient truss model Part II: Bar element stiffness matrices, International Review of Civil Engineering (accepted for publication).

Alsaleh M. I. (2004). "Numerical modelling of strain localisation in granular materials enhanced with micro-fabric properties" $\mathrm{PhD}$ thesis Louisiana State University.

B.S. Altan and E.C. Aifantis, On some aspects in the special theory of gradient elasticity, J. Mech. Behavior Mats. 8, 231-282 (1997).

Anderson W. B., Lakes, R. S. (1994). Size effects due to Cosserat elasticity and surface damage in closed-cell polymethacrylimide foam, J. Materials Science, 29, 6413-6419.

Argyris, Editor, (1978). Conferences on Finite Elements in Nonlinear Mechanics (FENOMECH), Stuttgart,

Argyris, Editor, (1981). Conferences on Finite Elements in Nonlinear Mechanics (FENOMECH), Stuttgart,

Argyris, Editor, (1994). Conferences on Finite Elements in Nonlinear Mechanics (FENOMECH), Stuttgart,

Askes H., Aifantis E. C. (2011). Gradient elasticity in statics and dynamics: An overview of formulations, length scale identification procedures, finite element implementations and new results. International Journal of Solids and Structures, 48, 13, pp. 1962-1990.

Bazant, Z. (1997). Fracturing truss model: Size effect in shear failure of reinforcement, J. of Engrg. Mech. ASCE. 123, 12761288.

Bazant, Z. P., Pijaudier-Cabot, G. (1989). Measurement of Characteristic Length of Nonlocal Continuum. J. of Engng. Mech., ASCE, V. 1 15, No. 4, pp. 755-767.

Begley, M.R., and Hutchinson, J.W. (1998). The mechanics of size-dependent indentation. J. Mech. Phys. Solids 46:2049-2068. 
Beheshti, A. (2017). Generalization of strain-gradient theory to finite elastic deformation for isotropic materials, Continuum Mechanics and Thermodynamics March, Volume 29, Issue 2, pp 493-507.

Ben-Amoz, M. (1976). A dynamic theory for composite materials. J Appl. Math. Ph. (ZAMP) 27, 83-99.

Beran, M., McCoy, J. (1970). Quart. Appl. Math. XXVIII, No. 2.

Bertram, A. (2015). Finite gradient elasticity and plasticity: a constitutive mechanical framework. Contin. Mech. Thermodyn. 27, 1039-1058.

Clemens, H., Mayer, S. and Scheu, C. (2017). Microstructure and Properties of Engineering Materials: From Fundamentals to Applications. Neutrons and Synchrotron Radiation in Engineering Materials Science: From Fundamentals to Applications: Second Edition https://onlinelibrary.wiley.com/doi/pdf/10.1002/9783527684489.ch1

Cosseret, E. Cosserat, F. (1909). Therie de Corps Deformables, Paris, (A. Hermann, ed.).

Dai, L.H., Bai, Y.L. (1998). J. Compos. Mater. 32246.

Dai, L.H., Ling, Z., Bai, Y.L. (2001). Compos. Sci. Technol. 61, 1057.

Dai, L.H., Liu, L.F., Bai Y.L. (2004). Effect of particle size on the formation of adiabatic shear band in particle reinforced metal matrix composites, Materials Letters, 58, 1773-1776.

Fleck, N.A., Muller, G.M., Ashby, M.F., Hutchinson, J.W. (1994). Strain gradient plasticity: theory and experiment. Acta. Metallurgica et Materialia 42:475-487, 1994. 9.

Gao, H., Huang, Y., and Nix, W.D. (1999). Modeling plasticity at the micrometer scale. Naturwissenschaften 86:507-515.

Geers. M.G.D., de Borst, R., Brekelmans, W.A.M, Peerlings, R.H.J. (1999). Validation and internal length scale determination for a gradient damage model: application to short glass-fibre-reinforced polypropylene. International Journal of Solids and Structures;36(17):2557-83.

Geol, H. C., Stojadinovic. B., Lee, K. H., (1997). Truss analogy for steel moment connection, AISC Engrg. J. $2^{\text {nd }}$ quarter, 43-53.

Greer, J. R., \& De Hosson, J. T. M. (2011). Plasticity in small-sized metallic systems: Intrinsic versus extrinsic size effect. Progress in Materials Science, 56(6), 654-724. http://doi.org/10.1016/j.pmatsci.2011.01.005

Han Q.H., Asgari, A., Hodgson, P. D. et al. (2014). Strain partitioning in dual-phase steels containing tempered martensite. Mater Sci Eng.;611:90-99.

Isasti, N., Jorge-Badiola, D., Taheri, M.L. et al. (2014). Microstructural Features Controlling Mechanical Properties in Nb-Mo Microalloyed Steels. Part I: Yield Strength. Metall and Mat Trans A 45, 4960-4971.

Kroner, E., (1967). Elasticity theory of materials with long range cohesive forces. Int. J. Solids Struct. 24, 581-597.

Lakes, R. S. (1995). Experimental methods for study of Cosserat elastic solids and other generalized continua, in Continuum models for materials with micro-structure, ed. H. Muhlhaus, J. Wiley, N. Y. Ch. 1, p. 1-22, (1995).

Lakes, R. S., (2001a). “Extreme Damping in Compliant Composites with a Negative-Stiffness Phase." Philosophical Magazine Letters 81(2): 95-100.

Lakes R. S., (2001b). Extreme Damping in Composite Materials with a Negative Stiffness Phase Physical Review Letters Vol. 86, (13), 2897-2900. DOI: 10.1103/PhysRevLett.86.2897.

Lam, D.C.C., Yang, F., Chong, A.C.M., Wang, J., Tong, P. (2003). "Experiments and theory in strain gradient elasticity", J. Mech. Phys. Solids, Vol. 51 pp.1477-508.

Lazar, M. (2016). Irreducible decomposition of strain gradient tensor in isotropic strain gradient elasticity. ZAMM 96, 129113052016.

Ling, Z., Luo, L., Dodd, B. (1994). J. Phys. IV 4453.

Lloyd, D.J. (1994), Particle reinforced aluminum and magnesium matrix composites. Int. Mater. Rev.39:1-23, 1994.

Lotti, C., Correa, C.A., Canevarolo, S.V. (2000). Mechanical and Morphological Characterization of Polypropylene Toughened with Olefinic Elastomer Materials Research, Vol. 3, No. 2, 37-44, 2000.

Mallick, P.K. (1997). Fiber Reinforced Composites. Materials, Manufacturing and Design; Marcel Dekker Inc.: New York, NY. 
McElhaney, K.W., Valssak, J.J., Nix, W.D. (1998). Determination of indenter tip geometry and indentation contact area for depth sensing indentation experiments. J. Mater. Res. 13:1300-1306.

Mindlin, R. D. (1964). Microstructure in linear elasticity, Arch. Ration. Mech. Anal., 16, 51-78.

Moore, B., Jaglinski, T., Stone, D. S., Lakes, R. S. (2006). Negative incremental bulk modulus in foams, Philosophical Magazine Letters, Vol. 86, No. 10, October, 651-659.

Nix, W.D., Gao, H., (1998). Indentation size effects in crystalline materials: a law for strain gradient plasticity. J. Mech. Phys. Solids $46,411-425$.

Paik, P., Kar, K. K. (2009) Polypropylene nanosphere: particle size and crystal structure, International Journal of Plastic Technology, 13:68.

Papadopoulos, P.G., Xenidis, H.C. (1998) Amount of Confinement Preventing Global Instability of a Concrete Column, Proc. 11 ${ }^{\text {th }}$ European Conference of Earthquake Engineering, Paris, Vol. 3.

Park, K., Nishiyama, M., Nakada, N., et al. (2014). Effect of the martensite distribution on the strain hardening and ductile fracture behaviors in dual-phase steel. Mater Sci Eng., 604:135-141.

Peerlings, R.H.J., Fleck N.A. (2004). Computational Evaluation of Strain Gradient Elasticity Constants International Journal for Multiscale Computational Engineering, 2(4)599-619.

Polizzotto, C. (2010). Strain gradient plasticity, strengthening effects and plastic limit analysis International Journal of Solids and Structures 47 100-112.

Polizzotto, C. (2003). Gradient elasticity and nonstandard boundary conditions. Int. J. of Solids and Structures, Vol. 40, No. 26, 12 , pp. 7399-7423(25).

Roylance D. (2001) Stress-Strain Curves: Department of Materials Science and Engineering, Massachusetts Institute of Technology, Cambridge August 23.

Ru, C.Q., Aifantis, E.C. (1993). A simple approach to solve boundary-value problems in gradientelasticity, Acta Mechanica, vol 10, pp. 59-68.

Shu, J.Y., Fleck, N.A. (1998) The prediction of a size effect in micro-indentation. Int. J. Solids Struct. 35:1363-1383.

Stolken, J.S., Evans, A.G. A. (1998). Microbend test method for measuring the plasticity lengthscale. Acta Mater. 46:51095115.

Sun, D.Z., Honig, A. (1994). Significance of the characteristic length for micromechanical modelling of ductile fracture Transactions on Engineering Sciences vol 6, WIT Press.

Tasan, C.C., Diehl, M., Yan, D., et al. (2014). Integrated experimental simulation analysis of stress and strain partitioning in multiphase alloys. Acta Mater.; 81:386-400.

Toupin, R. A. (1962). Theories of Elasticity with Couple-stresses, Arch. Rational Mech. Anal., vol. 17, pp. 85-112.

Tsagrakis, I., Aifantis, E.C. (2002). Recent developments in gradient plasticity-Part I: formulation and size effects. ASME Trans. J. Eng. Mat. Tech. 124:352-357.

Tsepoura, K. G., Papargyri-Beskou, S., Polyzos D., Beskos, D. E. (2002). Static and dynamic analysis of a gradient-elastic bar in tension, Achive of Applied Mechanics, 72, pp. $483-497$.

Uchic, M. D., Dimiduk, D. M., Florando, J. N., Nix, W. D. (2004). Sample Dimensions Influence Strength and Crystal Plasticity Science Vol 305, 13, August www.sciencemag.org

Umesh, B., Rajagopal A., Reddy, J. N., (2017). One dimensional nonlocal integro-differential model and gradient elasticity model: Approximate solutions and size effects, Mechanics of Advanced Materials and Structures, DOI: 10.1080/15376494.2017.1373313.

Voyiadjis G. Z., Song C. R. (2006), "The Coupled Theory of Mixtures in Geomechanics with Applications” (Springer, Berlin).

Wang, M.M., Tasan CC, Ponge, D., et al. (2015). Nanolaminate transformation-induced plasticity and twinning-induced plasticity steel with dynamic strain partitioning and enhanced damage resistance. Acta Mater. 85: 216-228. 
Wu, X.L., Yang, M.X., Yuan, F.P., et al. (2015). Heterogeneous lamella structure unites ultrafine-grain strength with coarse grain ductility. Proc Natl Acad Sci USA. 112: 14501- 14505.

Wu, X., Zhu Y., (2017) Heterogeneous materials: a new class of materials with unprecedented mechanical properties, Materials Research Letters, 5:8, 527-532, DOI: 10.1080/21663831.2017.1343208

Xi, Z., Zhu, J., Tang, H., Ao, Q., Zhi, Hao., Wang, J., Li, C. (2011).Progress of Application Researches of Porous Fiber Metals, Materials, 4, 816-824; doi:10.3390/ma4040816.

Yang, M.X., Pan, Y., Yuan, F.P., et al. (2016a). Back stress strengthening and strain hardening in gradient structure. Mater. Res. Lett. 4:145-151.

Yang M.X., Yuan F.P., Xie Q.G., et al. (2016b). Strain hardening in Fe16Mn-10Al-0.86C-5Ni high specific strength steel. Acta Mater.;109:213-222.

Yuan, H., Chen, J. (2001). Identification of the intrinsic material length in gradient plasticity theory from micro-indentation tests. Int. J. Solids Struct. 38:8171-8187.

Zou, S., Wan, Z., Lu, L., and Tang, Y. (2016). Experimental Study on Tensile Properties of a Novel Porous Metal Fiber/Powder Sintered Composite Sheet Materials, 9, 712; doi:10.3390/ma9090712. 